\title{
Guiding Evolutionary Multi-Objective Optimization with Generic Front Modeling
}

\author{
Ye Tian, Xingyi Zhang, Ran Cheng, Cheng He, and Yaochu Jin, Fellow, IEEE
}

\begin{abstract}
In evolutionary multi-objective optimization, the Pareto front is approximated using a set of representative candidate solutions with good convergence and diversity. However, most existing multi-objective evolutionary algorithms have general difficulty in the approximation of Pareto fronts with complicated geometries. To address this issue, we propose a generic front modeling method for evolutionary multi-objective optimization, where the shape of the nondominated front is estimated by training a generalized simplex model. On the basis of the estimated front, we further develop a multi-objective evolutionary algorithm, where both the mating selection and environmental selection are driven by the approximate non-dominated fronts modeled during the optimization process. For performance assessment, the proposed algorithm is compared with several state-of-the-art evolutionary algorithms on a wide range of benchmark problems with various types of Pareto fronts and different numbers of objectives. Experimental results demonstrate that the proposed algorithm performs consistently on a variety of multi-objective optimization problems.
\end{abstract}

Index Terms-Evolutionary algorithm, multi- and manyobjective optimization, front modeling, fitness function

\section{INTRODUCTION}

$\mathbf{M}$ ULTI-objective optimization problems (MOPs) often involve two or more conflicting objectives to be optimized simultaneously, which widely exist in real-world applications [1], [2]. When the number of objectives is larger than three, MOPs are also known as many-objective optimization problems (MaOPs) [3]. Since there does not exist a single solution that optimizes

Manuscript received -. This work was supported in part by the National Natural Science Foundation of China under Grant 61822301, Grant 61672033, Grant 61502004, and Grant 61502001, Anhui Provincial Natural Science Foundation for Distinguished Young Scholars under Grant 1808085J06, and Shenzhen Peacock Plan under Grant KQTD2016112514355531. The work of Y. Jin was supported in part by the U.K. EPSRC under Grant EP/M017869/1. (Corresponding authors: Xingyi Zhang and Ran Cheng)

Y. Tian is with the Institute of Physical Science and Information Technology, Anhui University, Hefei 230601, China (email: field910921@gmail.com).

$X$. Zhang is with the Institute of Bio-inspired Intelligence and Mining Knowledge, School of Computer Science and Technology, Anhui University, Hefei 230039, China (email: xyzhanghust@gmail.com).

$\mathrm{R}$. Cheng and $\mathrm{C}$. He are with the Shenzhen Key Laboratory of Computational Intelligence, University Key Laboratory of Evolving Intelligent Systems of Guangdong Province, Department of Computer Science and Engineering, Southern University of Science and Technology, Shenzhen 518055, China (email: ranchengcn@gmail.com; chenghehust@gmail.com).

Y. Jin is with the Department of Computer Science, University of Surrey, Guildford, Surrey, GU2 7XH, U.K. (email: yaochu.jin@surrey.ac.uk). all conflicting objectives, it is usually expected that a set of solutions will be obtained as trade-offs between different objectives. All the trade-off solutions, known as Pareto optimal solutions, constitute the Pareto set (PS), and the image of the PS in objective space is known as the Pareto front (PF) [4].

Over the last two decades, the multi-objective evolutionary algorithms (MOEAs) have demonstrated high effectiveness in solving MOPs [4]. In terms of the environmental selection strategies, the existing MOEAs can be roughly classified into three categories, i.e., Pareto dominance based MOEAs, decomposition based MOEAs, and indicator based MOEAs. The Pareto dominance based MOEAs utilize the non-dominated sorting approaches [5] to divide candidate solutions into several fronts at the first stage, and afterwards distinguish the candidate solutions in the same front by other diversity metrics [6], [7]. The decomposition based MOEAs are characterized by decomposing the original MOP into a number of single-objective optimization problems (SOPs) or simpler MOPs to be solved in a collaborative manner [8], [9]. As for the indicator based MOEAs, where the environmental selection is based on a performance indicator such as hypervolume [10], inverted generational distance [11], and R2 [12], the fitness of a candidate solution is measured by its contribution to the indicator value with respect to the whole population [13], [14].

As reported in some recent studies [15], [16], despite that evolutionary multi-objective optimization has been widely verified on a variety of benchmark problems, it is still a challenging task to maintain a good distribution of the candidate solutions on various types of irregular PFs. For example, in decomposition based MOEAs, since the predefined weight vectors are uniformly sampled on the unit hyperplane, the candidate solutions distributed in the middle of convex/concave PFs will be more/less crowded than those on the border, and such a phenomenon can become worse when the PF has a sharp peak or long tail [17]. Besides, since the distribution of the candidate solutions is mostly determined by the predefined weight vectors, the difference between the PF shape and the distribution of weight vectors could also lead to a substantial deterioration of the performance of decomposition based MOEAs [15]. Similar issues could also exist in indicator (e.g. hypervolume [10]) based MOEAs [13], [18], where the candidate solutions have 
biased distribution in the middle of a convex/concave PF due to their larger/smaller hypervolume contributions than those on the borders.

To address the above issues, some approaches were proposed to enhance the performance consistency of MOEAs for tackling MOPs with various PFs, for instance, the adaptive parameter setting based approaches [19], [20] and the weight vector adaptation based approaches [17], [21]. However, little work has been dedicated to direct modeling the geometrical structures of the PFs. Intuitively, if an MOEA is able to model the PF of a given MOP, the algorithm can ideally make selections among candidate solutions with respect to the modeled $\mathrm{PF}$, regardless of the specific PF shape. Motivated by this, we propose a generic front modeling based MOEA for enhancing the performance consistency of MOEAs in solving MOPs with various PFs, where the contributions of this paper can be summarized as follows:

1) A generic front modeling (GFM) method is proposed for estimating the PF of a given MOP by iteratively training a generalized simplex model. In the proposed GFM method, the non-dominated solutions obtained during the optimization process are used as the training data, and the LevenbergMarquardt algorithm is employed to estimate the parameters of the model by minimizing the training error. As demonstrated by the examples given in Section III-B, the proposed GFM can estimate various types of PFs with low errors.

2) An evolutionary algorithm (called GFM-MOEA) is developed on the basis of GFM, where both mating selection and environmental selection are driven by the estimated PF models. To be specific, a novel fitness function is proposed as the selection criterion, where the convergence quality of a candidate solution is measured by its distance to the estimated PF, while the diversity quality is measured by its projection on the estimated PF model. Experimental results demonstrate the effectiveness of the proposed GFM-MOEA on MOPs and MaOPs with various types of PFs.

The rest of this paper is organized as follows. In Section II, we briefly review some existing MOEAs for enhancing the performance consistency and MOEAs based on PF modeling. The proposed GFM method is elaborated in Section III, followed by the description of GFM-MOEA in Section IV. Experimental results and discussions are given in Section V. Lastly, conclusions are drawn in Section VI.

\section{RELATED WORK}

A. MOEAs for Enhancing the Performance Consistency in Solving MOPs with Various PFs

There are a large number of MOEAs developed to enhance the performance consistency in solving MOPs with various PFs [17], [19]-[25], which can be roughly grouped into the following three categories.
The first category is motivated to deal with scaled objectives by normalizing the objective values. For MOEAs in this category, the objective values of all the candidate solutions in the population are usually normalized according to the intercept of each axis and the hyperplane constructed by the extreme solutions [26]. NSGA-III [9], I-DBEA [27] and $\theta$-DEA [28], which show consistent performance on MOPs with badly-scaled PFs, are three representative MOEAs belonging to this category.

The second category aims to adapt the fitness function to match the PF shape. Take MOEA/D-PaS [20] as an example, where the performance of decomposition based MOEAs is enhanced using a Pareto adaptive scalarizing method. Specifically, MOEA/D-PaS uses a weighted $L_{p}$ scalarizing method as the aggregation function, where the weighted sum and Tchebycheff methods are special cases of $L_{p}$ when $p=1$ and $p=\infty$, respectively. Then the algorithm finds the $p$ value for each weight vector such that the optimal solution identified by the associated $L_{p}$ scalarizing method is the closest one to the weight vector. In this way, the contour curve of the $L_{p}$ scalarizing method can approximate the curvature of the true PF, and thus lead to the consistent performance on problems with various PF shapes.

The third category applies weight vector adaptation, e.g., A-NSGA-III [9], MOEA/D-AWA [17], and RVEA* [21], where the basic idea is to adjust the distribution of the weight vectors according to the candidate solutions in the current population or an external archive. Normally, the initial weight vector set has a simplexlike distribution, while the adapted weight vector set can have a distribution similar to the PF shape, hence enhancing the performance of the MOEAs to achieve the maximum coverage of the PF.

However, due to the various PF shapes of MOPs, most of the above algorithms have limitations in solving MOPs. On one hand, NSGA-III and MOEA/D-PaS only estimate the rough scales or curvatures of the PFs, which may not work on MOPs with complicated irregular PFs. On the other hand, despite that A-NSGA-III, MOEA/DAWA, and RVEA* are tailored for MOPs with irregular PFs, they fail to perform consistently on MOPs with regular PFs [25]. In contrast to the above MOEAs, there are some other MOEAs which enhance the performance consistency by building models to explicitly estimate the PFs [29]-[32]. In the next subsection, we briefly review some representative MOEAs in this category.

\section{B. MOEAs Based on Pareto Front Modeling}

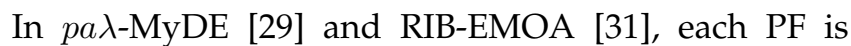
associated with one curve in the family:

$$
\left\{f_{1}^{p}+f_{2}^{p}+\ldots+f_{M}^{p}=1: 0 \leq f_{1}, \ldots, f_{M} \leq 1, p>0\right\},
$$

where $f_{i}$ denotes the $i$-th objective value and $M$ denotes the number of objectives. As can be observed from the equation, there is only one parameter $p$ in the model, which determines the curvature of the PF. 
More specifically, the PF becomes convex, concave, and linear when $p<1, p>1$, and $p=1$, respectively. To determine the value of $p, p a \lambda-\mathrm{MyDE}$ tries to minimize the difference between the hypervolume value of the PF model estimated using (1) and the hypervolume value of the non-dominated solutions in the population, while RIB-EMOA uses the maximum bulge in the nondominated solution set to approximate the PF curve.

In MMEA [32], the PF was estimated by an $M-1$ dimensional simplex, i.e.:

$$
a_{1} f_{1}+a_{2} f_{2}+\ldots+a_{M} f_{M}=1,
$$

where the estimated PF model is always linear. To determine the values of parameters $a_{1}, \ldots, a_{M}$, MMEA adopts a hyperplane by using the extreme points in the non-dominated set, then moves the hyperplane along its normal direction to a position such that no point on the hyperplane is dominated by any solution in the current population. The values of $a_{1}, \ldots, a_{M}$ are determined by the intercepts of the hyperplane on the axes.

Although these existing Pareto front modeling methods have been successfully applied in MOEAs, most of them have limitations in estimating various PFs, mainly due to the following two reasons. First, these models have very limited expression ability, such that they cannot be used for the modeling of PFs with complicated shapes. For example, since there is only one parameter controlling the curvature of PF in (1), the PF to be modeled should be symmetrical and well normalized. As for the model in (2), although it is able to express unnormalized PFs using parameters $a_{1}, \ldots, a_{M}$, the PF to be modeled should always be linear. Second, due to the high complexity of hypervolume calculations, the parameter optimization in these models is also challenging, especially when the number of objectives is large. In order to tackle problems with various types of PFs and different numbers of objectives, in this paper, we propose a generic Pareto front modeling method, called GFM, which adopts a generalized simplex model coupled with an effective training method for parameter optimization.

\section{The Proposed Generic PAReto Front MODELING METHOD}

\section{A. The Model in GFM}

In GFM, the PF shape of the MOP to be solved is estimated using the following model:

$$
\left\{\begin{array}{l}
a_{1}\left(f_{1}^{\prime}\right)^{p_{1}}+a_{2}\left(f_{2}^{\prime}\right)^{p_{2}}+\ldots+a_{M}\left(f_{M}^{\prime}\right)^{p_{M}}=1 \\
f_{1}^{\prime}, \ldots, f_{M}^{\prime} \geq 0, a_{1}, \ldots, a_{M}, p_{1}, \ldots, p_{M}>0
\end{array},\right.
$$

where $a_{i}$ and $p_{i}$ are parameters for controlling the scale and curvature of the PF in terms of the $i$-th objective, respectively, $M$ denotes the number of objectives, and $f_{i}^{\prime}=f_{i}-z_{i}^{*}$ is the translated value of the $i$-th objective by being subtracted by the ideal point ${ }^{1}$. It can be observed that the image of (3) is always in the first quadrant and

\footnotetext{
${ }^{1}$ For a minimization problem, the ideal point is a vector that consists of the minimum value of each objective function.
}
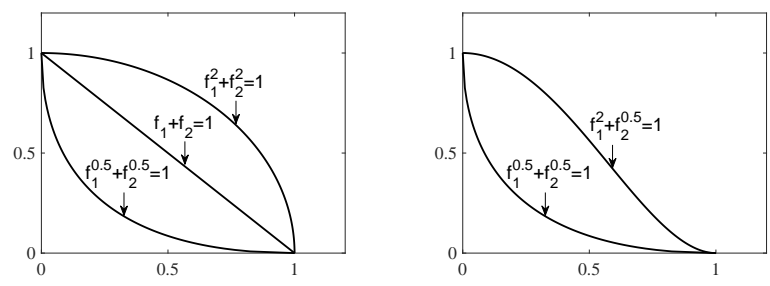

Fig. 1. The effectiveness of $p_{i}$ in controlling the shape of the PF $f_{1}^{p_{1}}+f_{2}^{p_{2}}=1$, where the PF is convex, concave and linear when $p_{i}<1$, $p_{i}>1$, and $p_{i}=1$, respectively. In addition, the PF is symmetrical or asymmetrical when $p_{i}$ have the same or different values, respectively.

intersects all the axes. However, since the PFs of some MOPs do not intersect all the axes (e.g., DTLZ7 [33]), the objective values are translated by subtracting the ideal point to make the PFs consistent with (3) [21]. It is worth noting that in some recent studies [17], [34], the objective values are suggested to be subtracted by a point lower than the ideal point, i.e., $f_{i}^{\prime}=f_{i}-\left(z_{i}^{*}-\epsilon_{i}\right)$ with $\epsilon_{i}>$ 0 . However, according to the experimental results given in Section V-F, a better performance is achieved in the proposed GFM when $\epsilon_{i}=0$.

The model in (3) is a generalization of (1) and (2), which has better ability in expressing complicated PF shapes. In contrast to the modes in (1) and (2), which can only approximate symmetrical PFs or linear PFs, the proposed model in (3) can approximate convex, concave and linear PFs in terms of the $i$-th objective when $p_{i}<1, p_{i}>1$, and $p_{i}=1$, respectively. In addition, the PFs become symmetrical or asymmetrical when $p_{i}$ have the same or different values, respectively. In addition, while the PF to be modeled by (1) should be well normalized, the proposed model in (3) is capable of modeling PFs with arbitrarily scaled PFs due to the parameters $a_{1}, \ldots, a_{M}$. Fig. 1 depicts five PFs obtained by using the model in (3) with different settings of $p_{1}, \ldots, p_{M}$, which clearly confirms the effectiveness of $p_{i}$ in controlling the shape of the estimated PF. As further illustrations to the properties of the model, we have the following two propositions.

Proposition 1: All of the points sampled on the surface generated by (3) are mutually non-dominated.

Proof: Given a point $\mathbf{x}_{1}$ arbitrarily sampled on the surface generated by (3), for any point $\mathbf{x}_{2}$ which is dominated by $\mathrm{x}_{1}$, it satisfies:

$$
\left\{\begin{array}{l}
\forall i \in 1, \ldots, M: a_{i} f_{i}^{p_{i}}\left(\mathbf{x}_{1}\right) \leq a_{i} f_{i}^{p_{i}}\left(\mathbf{x}_{2}\right) \\
\exists j \in 1, \ldots, M: a_{j} f_{j}^{p_{j}}\left(\mathbf{x}_{1}\right)<a_{j} f_{j}^{p_{j}}\left(\mathbf{x}_{2}\right)
\end{array},\right.
$$

where $a_{1}, \ldots, a_{M}, p_{1}, \ldots, p_{M}>0$ and $f_{i}\left(\mathbf{x}_{1}\right)$ denotes the $i$-th objective value of point $\mathbf{x}_{1}$. Then we have:

$$
\Sigma_{i=1}^{M} a_{i} f_{i}^{p_{i}}\left(\mathbf{x}_{2}\right)>\Sigma_{i=1}^{M} a_{i} f_{i}^{p_{i}}\left(\mathbf{x}_{1}\right)=1 .
$$

Therefore, $\mathbf{x}_{2}$ cannot be on the surface generated by (3). Analogously, for any point $\mathbf{x}_{3}$ which dominates $\mathbf{x}_{1}$, it satisfies:

$$
\sum_{i=1}^{M} a_{i} f_{i}^{p_{i}}\left(\mathbf{x}_{3}\right)<\Sigma_{i=1}^{M} a_{i} f_{i}^{p_{i}}\left(\mathbf{x}_{1}\right)=1,
$$


which indicates that $\mathbf{x}_{3}$ cannot be on the surface generated by (3) either. Therefore, all the points on the surface generated by (3) can neither be dominated by $\mathbf{x}_{1}$ nor dominate $\mathbf{x}_{1}$, i.e., they are mutually non-dominated.

Proposition 2: The model in (3) satisfies the strictly increasing sufficiency property ${ }^{2}$ [35] , i.e., the function of the model is strictly increasing on each objective.

Proof: Let $G=a_{1} f_{1}^{p_{1}}+a_{2} f_{2}^{p_{2}}+\ldots+a_{M} f_{M}^{p_{M}}-1$, according to (3), we have

$$
\frac{\partial G}{\partial f_{i}}=a_{i} p_{i} f_{i}^{p_{i}-1}>0, \forall i \in 1, \ldots, M,
$$

where $f_{1}, \ldots, f_{M}, a_{1}, \ldots, a_{M}, p_{1}, \ldots, p_{M}>0$, hence $G$ is a strictly increasing function on each objective $f_{i}$.

The above propositions support that the proposed model in (3) has good capability in providing a comprehensive outline of the estimated PF shape and thus guides the search process towards promising directions, where more details will be demonstrated in Section IV.

\section{B. The Training Method in GFM}

In the proposed GFM, the training process is performed by minimizing the error of the model with respect to the non-dominated solutions by the LevenbergMarquardt algorithm [36], which is commonly seen in the training of feedforward neural networks. The reason that we use Levenberg-Marquardt algorithm is due to the fact that it is more efficient than other gradient based techniques in training models with a few parameters [37]. To begin with, a set of non-dominated solutions is collected from the current population and translated to be the training data, where the training error of the $i$-th non-dominated solution can be calculated as:

$$
e_{i}=\sum_{j=1}^{M} a_{j}\left(f_{i j}^{\prime}\right)^{p_{j}}-1,
$$

and consequently, the mean squared error (MSE) of the whole training set is

$$
M S E=\frac{1}{N} \sum_{i=1}^{N} e_{i}^{2},
$$

where $M$ is the number of objectives and $N$ is the number of non-dominated solutions. Then we have

$$
\frac{\partial e_{i}}{\partial a_{j}}=\left(f_{i j}^{\prime}\right)^{p_{j}}
$$

and

$$
\frac{\partial e_{i}}{\partial p_{j}}=a_{j}\left(f_{i j}^{\prime}\right)^{p_{j}} \ln f_{i j}^{\prime}
$$

Thus the Jacobian matrix $J$ can be formulated as:

$$
J=\left[\begin{array}{cccccc}
\frac{\partial e_{1}}{\partial a_{1}} & \cdots & \frac{\partial e_{1}}{\partial a_{M}} & \frac{\partial e_{1}}{\partial p_{1}} & \cdots & \frac{\partial e_{1}}{\partial p_{M}} \\
\vdots & \ddots & \vdots & \vdots & \ddots & \vdots \\
\frac{\partial e_{N}}{\partial a_{1}} & \cdots & \frac{\partial e_{N}}{\partial a_{M}} & \frac{\partial e_{N}}{\partial p_{1}} & \cdots & \frac{\partial e_{N}}{\partial p_{M}}
\end{array}\right],
$$

\footnotetext{
${ }^{2}$ According to the strictly increasing sufficiency, let $G(\mathbf{f}): \mathbb{R}^{M} \rightarrow \mathbb{R}$ be a strictly increasing function on each coordinate, let $F=\left\{\mathbf{f} \in \mathbb{R}^{M}\right.$ | $G(\mathbf{f})=0\}$, then $F$ is an estimated PF.
}

and the steps for updating the parameters are

$$
\Delta=-\left[J^{T} J+\lambda \operatorname{diag}\left(J^{T} J\right)\right]^{-1} J^{T} e,
$$

where $e=\left(e_{1}, \ldots, e_{N}\right)^{T}$ and $\lambda$ is the damping factor, which is dynamically adjusted during the iterations. Finally, the parameters $a_{j}$ and $p_{j}$ can be updated as follows:

$$
\left\{\begin{array}{l}
a_{j}=a_{j}+\Delta_{j} \\
p_{j}=p_{j}+\Delta_{j+M}
\end{array},\right.
$$

where $\Delta_{j}$ denotes the $j$-th element of vector $\Delta$. The above procedure will be iterated until the MSE cannot be decreased.

In order to verify the approximation capability of the proposed GFM, we apply it to the estimation of the PF models of several representative MOPs, i.e., DTLZ1, DTLZ2, DTLZ5, DTLZ7 [33], convex DTLZ2 (CDTLZ2) [9], and inverted DTLZ2 (IDTLZ2) [24] with 3 and 10 objectives, respectively. A number of 100 points on the $\mathrm{PF}$ of each test instance are sampled as the training set. Table I lists the true PF, the estimated PF and the final MSE on each test instance, where the MSE is calculated using approximately 10000 points sampled on the true PF. For better observation, Fig. 2 shows the modeling results on all the test instances with 3 objectives.

As evidenced by the results in Table I, the proposed GFM is capable of modeling the PFs of all the 12 test instances with low estimation errors with regard to the test data sampled on the true PFs, no matter whether the PF is linear (DTLZ1), concave (DTLZ2), convex (CDTLZ2), degenerated (DTLZ5), disconnected (DTLZ7) or even inverted (IDTLZ2). Since the PFs of DTLZ1, DTLZ2, and CDTLZ2 are simple and regular, GFM has obtained the estimated PFs which are almost the same as the true PFs.

As for the problems such as DTLZ5, DTLZ7, and IDTLZ2 which have irregular PFs, the estimated PFs are still able to cover the true PFs, although the shapes are not exactly matched. For example, as shown in Fig. 3, although the PF estimated by GFM is different from the true PF of DTLZ7, the estimated PF is still able to very well fit the non-dominated parts of the true PF. As will be demonstrated in the following section, such an estimated PF model can be used to guide the search towards the true PF and meanwhile maintain proper population diversity, which is particularly desirable in the designs of MOEAs.

\section{The Proposed GFM-MOEA}

\section{A. The GFM Based Fitness Function}

The GFM based fitness function is the key component in GFM-MOEA, where the fitness of the candidate solutions in the same non-dominated front is measured according to the estimated PF modeled by GFM. Specifically, the fitness of a candidate solution is measured by two criteria, the convergence criterion and the diversity criterion. As indicated by the Proposition 2 in Section III-A, since the estimated PF is strictly increasing on each objective, the line connecting the objective vector of a 
TABLE I

Estimation RESUlts of GFM ON DTLZ1, DTLZ2, DTLZ5, DTLZ7, CDTLZ2, AND IDTLZ2 With 3 AND 10 OBJECTIVES.

\begin{tabular}{ccccc}
\hline Problem & $M$ & True PF & Estimated PF & MSE on Test Set \\
\hline DTLZ1 & 3 & $2 f_{1}+2 f_{2}+2 f_{3}=1$ & $1.9 \mathrm{e}-13$ \\
\hline DTLZ2 & 3 & $f_{1}^{2}+f_{2}^{2}+f_{3}^{2}=1$ & $2 f_{1}+2 f_{2}+2 f_{3}=1$ & $5.5 \mathrm{e}-15$ \\
\hline DTLZ5 & 3 & $2 f_{1}^{2}+f_{3}^{2}=1$ & $f_{1}^{2}+f_{2}^{2}+f_{3}^{2}=1$ & $2.7 \mathrm{e}-32$ \\
& & $f_{1}=f_{2}$ & $f_{1}^{2}+f_{2}^{2}+f_{3}^{2}=1$ & $2.9 \mathrm{e}-3$ \\
\hline DTLZ7 & 3 & $f_{1}\left[1+\sin \left(3 \pi f_{1}\right)\right]+f_{2}\left[1+\sin \left(3 \pi f_{2}\right)\right]+f_{3}=6$ & $0.85 f_{1}^{4.36}+0.85 f_{2}^{4.36}+0.5\left(f_{3}-2.61\right)^{0.61}=1$ & $6.9 \mathrm{e}-15$ \\
\hline CDTLZ2 & 3 & $f_{1}^{0.5}+f_{2}^{0.5}+f_{3}=1$ & $f_{1}^{0.5}+f_{2}^{0.5}+f_{3}=1$ & $2.1 \mathrm{e}-4$ \\
\hline IDTLZ2 & 3 & $\left(1-f_{1}\right)^{2}+\left(1-f_{2}\right)^{2}+\left(1-f_{3}\right)^{2}=1$ & $0.49 f_{1}^{0.48}+0.49 f_{2}^{0.48}+0.49 f_{3}^{0.48}=1$ & $5.0 \mathrm{e}-12$ \\
\hline \hline DTLZ1 & 10 & $\sum_{i=1}^{M} 2 f_{i}=1$ & $\sum_{i=1}^{M} 2 f_{i}=1$ & $5.4 \mathrm{e}-13$ \\
\hline DTLZ2 & 10 & $\sum_{i=1}^{M} f_{i}^{2}=1$ & $\sum_{i=1}^{M} f_{i}^{2}=1$ & $1.1 \mathrm{e}-30$ \\
\hline DTLZ5 & 10 & $f_{i}=(\sqrt{2} / 2)^{M-\max (i, 2)} \cos (0.5 \pi \theta), i<M$ & $0.85 f_{1}^{2}+0.85 f_{2}^{2}+0.84 f_{3}^{2}+0.82 f_{4}^{2}+0.81 f_{5}^{2}+$ & \\
\hline DTLZ7 & 10 & $f_{M}=\sin (0.5 \pi \theta), i=M$ & $0.81 f_{6}^{2}+0.83 f_{7}^{2}+0.92 f_{8}^{2}+1.13 f_{9}^{2}+f_{10}^{2}=1$ & \\
\hline CDTLZ2 & 10 & $f_{M}=2 M-\sum_{i=1}^{M-1} f_{i}\left[1+\sin \left(3 \pi f_{i}\right)\right]$ & $\sum_{i=1}^{M-1} 0.13 f_{i}^{1.13}+0.07\left(f_{M}-4.76\right)=1$ & $\sum_{i=1}^{M-1} f_{i}^{0.5}+f_{M}=1$ \\
\hline IDTLZ2 & 10 & $\sum_{i=1}^{M-1} f_{i}^{0.5}+f_{M}=1$ & $\sum_{i=1}^{M} 0.11 f_{i}^{0.56}=1$ & $1.1 \mathrm{e}-11$ \\
\hline
\end{tabular}
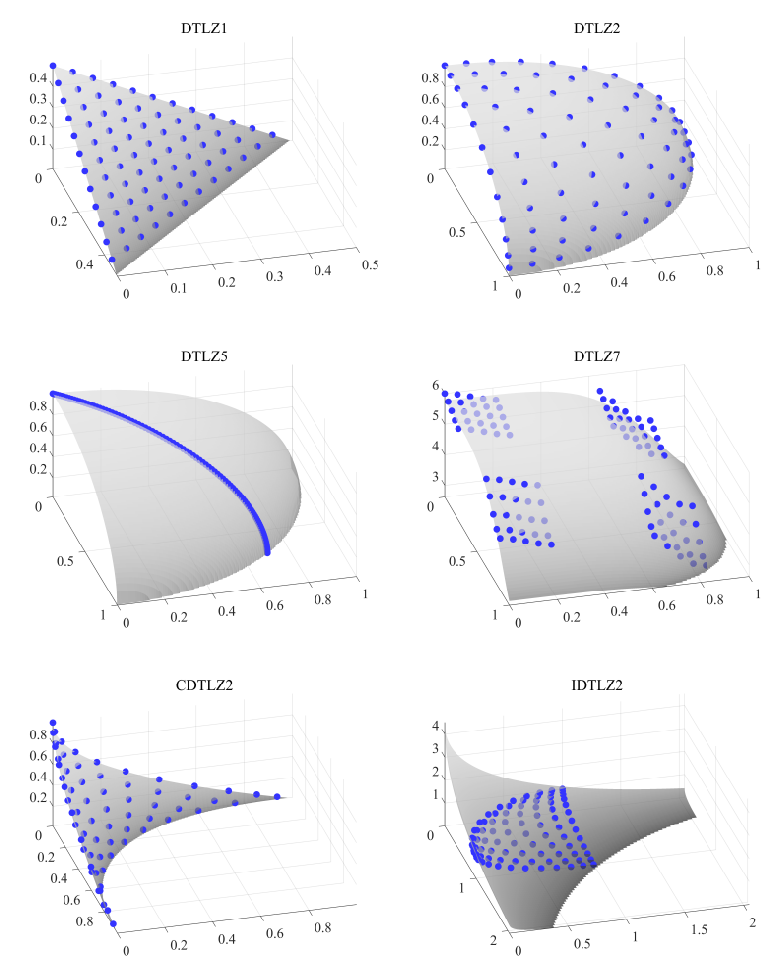

Fig. 2. The PFs models estimated by GFM on 3-objective DTLZ1, DTLZ2, DTLZ5, DTLZ7, CDTLZ2, and IDTLZ2, where the dots are the data points in the training sets, and the surfaces are the estimated PFs.

candidate solution $\mathbf{x}$ and the ideal point $\mathbf{z}^{*}$ has one and only one intersection point $\mathbf{y}$ on the estimated PF:

$$
\mathbf{y}=\left(r\left(f_{1}(\mathbf{x})-z_{1}^{*}\right)+z_{1}^{*}, \ldots, r\left(f_{M}(\mathbf{x})-z_{M}^{*}\right)+z_{M}^{*}\right)
$$

where the parameter $r$ is determined by

$$
a_{1}\left[r\left(f_{1}(\mathbf{x})-z_{1}^{*}\right)\right]^{p_{1}}+\ldots+a_{M}\left[r\left(f_{M}(\mathbf{x})-z_{M}^{*}\right)\right]^{p_{M}}=1
$$

On the basis of the intersection point $\mathbf{y}$, the convergence criterion of candidate solution $\mathrm{x}$ is defined as its
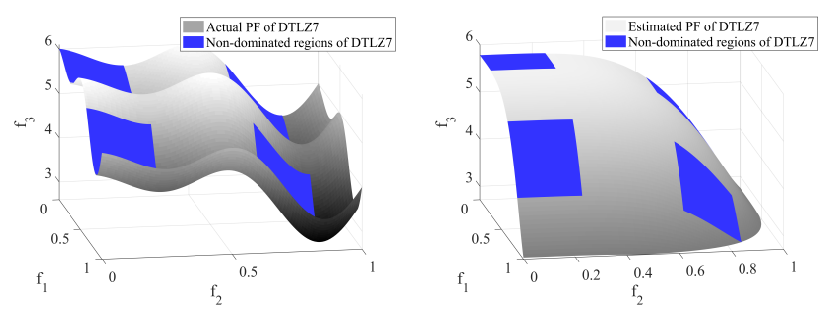

Fig. 3. The true PF and the estimated PF of 3-objective DTLZ7.

distance to the intersection point associated with it:

$$
c(\mathbf{x})=\left\|\mathbf{y}-\mathbf{z}^{*}\right\|-\left\|\mathbf{f}(\mathbf{x})-\mathbf{z}^{*}\right\|,
$$

where $\|\cdot\|$ is the $L_{2}$-norm. The diversity criterion of candidate solution $\mathrm{x}$ is calculated by the distance from the intersection point to its nearest neighbor:

$$
d(\mathbf{x})=\left\|\mathbf{y}-\mathbf{y}^{\prime}\right\|,
$$

where $\mathbf{y}^{\prime}$ denotes the nearest intersection point to $\mathbf{y}$ among all the intersection points of the remaining candidate solutions in the same non-dominated front.

With the convergence criterion $c(\mathbf{x})$ and the diversity criterion $d(\mathbf{x})$, the fitness of candidate solution $\mathbf{x}$ is further calculated as:

$$
\text { fitness }(\mathbf{x})=\theta \times c(\mathbf{x})+(1-\theta) \times d(\mathbf{x}),
$$

where $0<\theta<1$ is a predefined penalty parameter. In this work, $\theta=0.2$ is adopted in all cases, and the sensibility analysis of $\theta$ can be found in Section V-E.

\section{B. Procedure of GFM-MOEA}

For simplicity, the proposed GFM-MOEA (i.e., the MOEA based on GFM) adopts the same framework as NSGA-II [6], where the only difference is that the crowding distance in NSGA-II has been replaced by the GFM based fitness function fitness $(\mathbf{x})$ as introduced in the previous subsection. 


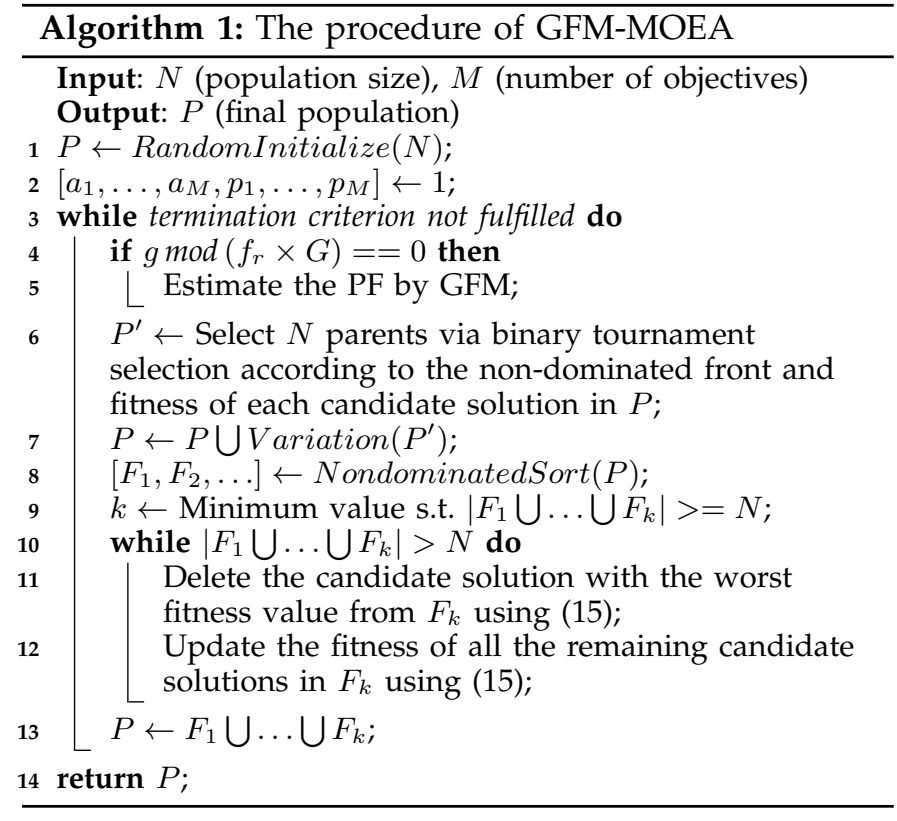

The pseudocode of the proposed GFM-MOEA is given in Algorithm 1. To begin with, an initial population with size $N$ is randomly generated, and the parameters $a$ and $p$ in GFM are initialized to the value of 1 . At each generation, GFM is used to model the approximate PF using the non-dominated solutions in the current population as training data. For the sake of stability and efficiency, GFM is employed every $f_{r} \times G$ generations, where $g$ denotes the current generation number, $G$ denotes the maximum number of generations, and $f_{r}$ is the parameter controlling the frequency. In this work, $f_{r}=0.1$ is adopted in all cases, and the sensibility analysis of $f_{r}$ is given in Section V-E.

Afterwards, the mating selection is performed for selecting $N$ parents from the current population via the binary tournament selection. More specifically, two candidate solutions are randomly picked up from the population each time, and the one having a smaller non-dominated front number will be selected as a parent. If the two candidate solutions are in the same non-dominated front, the candidate solution with better (larger) fitness value will be selected as a parent. After the $N$ parents are selected, the same number of offsprings are reproduced and merged to the population.

To perform environmental selection, the nondominated sorting is first performed to divide the merged population into several non-dominated fronts $F_{1}, F_{2}, \ldots$, where ENS-SS [5] is employed for MOPs and T-ENS [38] is employed for MaOPs. Then, the candidate solution $p$ with the worst (least) fitness value is deleted from $F_{k}$ one by one, where $F_{k}$ is the last front satisfying $\left|F_{1} \bigcup \ldots \bigcup F_{k}\right|>=N$, until $\left|F_{1} \bigcup \ldots \bigcup F_{k}\right|=N$ is reached. It is worth noting that, since the fitness value of each candidate solution is related to those of the others left in the current population, the fitness values need to be updated after each $p$ is deleted.

\section{Time Complexity of GFM-MOEA}

The computational cost mainly results from three operations in GFM-MOEA, namely, GFM, non-dominated sorting, and environmental selection. In GFM, the most time-consuming operation is the calculation of $\Delta$ in (9). Since the size of $J$ is $N \times 2 M$ with $N$ denoting the population size and $M$ denoting the number of objectives, the calculation of $J^{T} J$ has a time complexity of $O\left(M^{2} N\right)$, and the matrix inversion has a time complexity of $O\left(M^{3}\right)$. Hence, the time complexity of GFM is $O\left(G^{\prime} M^{2}(M+N)\right)$, where $G^{\prime}$ denotes the number of iterations of the Levenberg-Marquardt algorithm. By adopting ENS, the time complexity of non-dominated sorting is $O\left(M N^{2}\right)$ in the worst case [39]. In environmental selection, the time complexity for calculating the fitness of all the candidate solutions is $O\left(M N^{2}\right)$ according to (15), and the time complexity for updating the fitness of remaining solutions is $O\left(N^{3}\right)$ for deleting at most $N$ candidate solutions. Hence, the time complexity of environmental selection is $O\left(N^{2}(M+N)\right)$.

To summarize, suppose that the total number of generations is $G$ and the frequency of employing GFM is $f_{r}$, the overall time complexity of GFM-MOEA is $O\left(f_{r} G G^{\prime} M^{2}(M+N)\right)+O\left(G M N^{2}\right)+O\left(G N^{2}(M+N)\right)=$ $O\left(G N^{3}\right)$, with the assumption $f_{r} G^{\prime} \ll G$ and $M \ll N$.

\section{EMPIRICAL STUdies}

In this section, we first compare the proposed GFMMOEA with its three variants to verify the effectiveness of the GFM method in guiding the evolutionary multiobjective optimization process. Then, the performance of GFM-MOEA is assessed by the experimental comparisons with four popular MOEAs on several benchmark MOPs, and the experimental comparisons with four popular MOEAs on several benchmark MaOPs. Finally, the sensibility analysis of the parameters $\theta$ and $f_{r}$ in GFM-MOEA is presented, and the influence of the ideal point on the performance of GFM-MOEA is studied. All the experiments are performed on the MATLAB platform for evolutionary multi-objective optimization [40].

\section{A. Experimental Settings}

1) Compared MOEAs: Apart from the proposed GFMMOEA, four classical MOEAs are involved in the experiments on MOPs, namely, NSGA-II [6], MOEA/D [8], IBEA [41], and MOEA/D-AWA [17], which belong to Pareto dominance based MOEAs, decomposition based MOEAs, indicator based MOEAs, and decomposition based MOEAs, respectively. In particular, MOEA/DAWA is a variant of MOEA/D tailored for MOPs with complex PFs. Four recently proposed MOEAs are used in the experiment on MaOPs, namely, MOEA/DD [42], RVEA [21], MOEA/D-PaS [20], and VaEA [43], all of which have been verified to be effective in tackling MaOPs. In MOEA/D, MOEA/D-AWA, MOEA/DD, and MOEA/D-PaS, the size of neighborhood $T$ is set to $\lceil 0.1 N\rceil$, the neighborhood selection probability $\delta$ is set to 
0.9 , and the maximum number of solutions replaced by each offspring $n_{r}$ is set to $\lceil 0.01 N\rceil$, with $N$ denoting the population size. In addition, the Tchebycheff approach with transformed reference vectors [44] is utilized as the scalarization approach in MOEA/D. For MOEA/DAWA, the ratio of updated weight vectors is set to 0.05 , the ratio of iterations to evolve with only MOEA/D is set to 0.8 , and the generation interval of utilizing AWA is set to 5 for DTLZ1, DTLZ3, IDTLZ1, WFG1, WFG2, IWFG1, IWFG2, and MaF1-MaF15, and 2 for the rest problems. For IBEA, the fitness scaling factor $\kappa$ is set to 0.05 . For RVEA, the penalty parameter $\alpha$ in APD is set to 2, and the parameter $f_{r}$ controlling the frequency of reference vector adaption is set to 0.1 . The penalty parameter $\theta$ and the frequency $f_{r}$ of employing GFM in GFM-MOEA are set to 0.2 and 0.1 , respectively.

2) Test problems: 43 widely used multi-objective benchmark problems are used as the test problems, i.e., ZDT1-ZDT4, ZDT6 [45], DTLZ1-DTLZ7 [33], convex DTLZ2 (CDTLZ2) [9], inverted DTLZ1-DTLZ2 (IDTLZ1IDTLZ2) [24], WFG1-WFG9 [46], inverted WFG1-WFG4 (IWFG1-IWFG4), and MaF1-MaF15 [16]. Note that the IWFG1-IWFG4 are designed by a similar method to IDTLZ1 [24]. The length of decision variables of ZDT1ZDT3 is set to 30, and the length of decision variables of ZDT4 and ZDT6 is set to 10. For all the DTLZ problems, the length of decision variables is set to $K+M-1$, where $M$ denotes the number of objectives, and $K$ is set to 5 for DTLZ1 and IDTLZ1, 20 for DTLZ7, and 10 for the others. As for all the WFG problems, the length of decision variables is set to $K+L$, where $K$ and $L$ are set to $M-1$ and 10 respectively. The lengths of decision variables for the MaF problems are referred to [16]. The maximum number of generations is adopted as the termination criterion for all compared MOEAs, which is set to 500 for DTLZ1, DTLZ3, IDTLZ1, WFG1, WFG2, IWFG1, IWFG2, and MaF1-MaF15, and 200 for all the other problems.

3) Population sizing: The population size of all the compared MOEAs is set to the same on each MOP, namely, 100, 105, 126 and 275 for 2-, 3-, 5- and 10-objective MOPs, respectively. Accordingly, in MOEA/D, MOEA/D-AWA, MOEA/DD, RVEA, and MOEA/D-PaS, the parameters $\left(p_{1}, p_{2}\right)$ controlling the number of reference vectors along the outer and inner layers [9] are set to $(99,0),(13,0)$, $(5,0)$ and $(3,2)$ for $2,3,5$ and 10 objectives, since the number of reference vectors needs to be consistent with the population size in these algorithms.

4) Genetic operators: The simulated binary crossover (SBX) [47] and polynomial mutation [48] are employed in all the compared MOEAs for creating offsprings, and the parameter settings of them are identical in all the MOEAs for fair comparison. To be specific, the probability of crossover is set to 1 , the probability of mutation is set to $1 / D$, and the distribution index of both SBX and polynomial mutation is set to 20 , where $D$ denotes the length of decision variables.

5) Performance metrics: The inverted generational dis- tance (IGD) [49] and hypervolume (HV) [10] are employed to assess the performance of the compared MOEAs, both of which can measure the convergence and diversity of the results simultaneously. A smaller value of IGD indicates a better quality of the result, while a larger value of HV signals a better quality. In the calculation of IGD, roughly 10000 points uniformly sampled on the true PF of each test instance are adopted as the reference points. The detailed sampling method for each test instance can be found in [50]. As for the calculation of $\mathrm{HV}$, the reference point is set to $(1, \ldots, 1)$, and the objective values are normalized by the point $1.1 \times z^{\text {nad }}$ before the calculation, where $z^{\text {nad }}$ denotes the nadir point of the true PF. All the experiments are performed for 30 runs independently, and the mean value and the standard deviation of each result are recorded. Furthermore, the results are also analyzed by the Wilcoxon rank sum test with a significance level of 0.05 , where ' $+{ }^{\prime},{ }^{\prime}{ }^{\prime}{ }^{\prime}$ and ' $\approx$ ' indicate that the result of other MOEA is significantly better, significantly worse and statistically similar to that of the proposed GFMMOEA, respectively.

\section{B. Effectiveness of GFM}

To verify whether GFM is able to enhance the performance consistency of MOEAs in solving MOPs with different types of PFs, GFM-MOEA is compared with three of its variants, in which the parameters $a$ and $p$ are fixed to several specific values instead of being learned in the training of GFM. The DTLZ2 and CDTLZ2 are used as the test problems in the experiment, where DTLZ2 has a concave PF and CDTLZ2 has a convex PF.

For the first variant of GFM-MOEA, we make its parameters $a$ and $p$ identical with those in the true PF of DTLZ2, i.e., $a_{1}=\ldots=a_{M}=1$ and $p_{1}=\ldots=p_{M}=2$, as shown in Table I. Analogically, we make the parameters $a$ and $p$ of the second variant identical with those in the true PF of CDTLZ2, where $a_{1}=\ldots=a_{M}=1, p_{1}=\ldots=$ $p_{M-1}=0.5$ and $p_{M}=1$. As for the third variant, we set $a_{1}=\ldots=a_{M}=1$ and $p_{1}=\ldots=p_{M}=1$. For simplicity, the three variants of GFM-MOEA are hereafter denoted as GFM-MOEA ${ }_{D T L Z 2}$, GFM-MOEA ${ }_{C D T L Z 2}$, and GFM$\mathrm{MOEA}_{1}$, respectively.

The statistical results of HV values of GFM-MOEA and its three variants on DTLZ2 and CDTLZ2 are given in Table II, and the non-dominated solution sets with the median HV obtained by the four MOEAs on the problems with 3 objectives are plotted in Fig. 4. From Table II and Fig. 4, the following observations can be obtained. First, GFM-MOEA achieves a competitive performance on both DTLZ2 and CDTLZ2 in comparison with the three variants, which indicates the effectiveness of the proposed GFM in capturing different PF geometries. The GFM-MOEA demonstrates a similar performance with GFM-MOEA $_{D T L Z 2}$ on DTLZ2, and similar performance with GFM-MOEA ${ }_{C D T L Z 2}$ on CDTLZ2. Secondly, since the concave model used by GFM-MOEA ${ }_{D T L Z 2}$ is always 
TABLE II

Statistical Results of HV Values ObTained by GFM-MOEA and its Three VARIANTS On DTLZ2 AND CDTLZ2 With 3,5 AND 10 OBJECTIVES.

\begin{tabular}{cccccc}
\hline Problem & $M$ & GFM-MOEA & GFM-MOEA $_{D T L Z 2}$ & GFM-MOEA $_{C D T L Z 2}$ & GFM-MOEA $_{1}$ \\
\hline \multirow{2}{*}{ DTLZ2 } & 3 & $5.6112 \mathrm{e}-1(6.54 \mathrm{e}-4)$ & $5.6097 \mathrm{e}-1(1.00 \mathrm{e}-3) \approx$ & $4.8312 \mathrm{e}-1(1.11 \mathrm{e}-2)-$ & $5.4676 \mathrm{e}-1(1.45 \mathrm{e}-3)-$ \\
& 5 & $7.8353 \mathrm{e}-1(2.92 \mathrm{e}-3)$ & $7.8429 \mathrm{e}-1(2.49 \mathrm{e}-3) \approx$ & $7.0634 \mathrm{e}-1(1.77 \mathrm{e}-2)-$ & $7.7874 \mathrm{e}-1(2.82 \mathrm{e}-3)-$ \\
& 10 & $9.5536 \mathrm{e}-1(2.39 \mathrm{e}-3)$ & $9.5220 \mathrm{e}-1(1.75 \mathrm{e}-3)-$ & $9.1751 \mathrm{e}-1(3.62 \mathrm{e}-2)-$ & $9.2625 \mathrm{e}-1(5.89 \mathrm{e}-2) \approx$ \\
\hline \multirow{2}{*}{ CDTLZ2 } & 3 & $9.6354 \mathrm{e}-1(1.57 \mathrm{e}-4)$ & $9.2666 \mathrm{e}-1(7.25 \mathrm{e}-3)-$ & $9.6343 \mathrm{e}-1(1.75 \mathrm{e}-4) \approx$ & $9.4166 \mathrm{e}-1(6.11 \mathrm{e}-3)-$ \\
& $5.9919 \mathrm{e}-1(3.92 \mathrm{e}-5)$ & $9.9089 \mathrm{e}-1(4.11 \mathrm{e}-3)-$ & $9.9917 \mathrm{e}-1(4.14 \mathrm{e}-5) \approx$ & $9.9018 \mathrm{e}-1(1.46 \mathrm{e}-3)-$ \\
\multicolumn{2}{c}{10} & $1.0000 \mathrm{e}+0(5.79 \mathrm{e}-7)$ & $9.9987 \mathrm{e}-1(1.35 \mathrm{e}-4)-$ & $1.0000 \mathrm{e}+0(2.67 \mathrm{e}-7) \approx$ & $9.9969 \mathrm{e}-1(1.55 \mathrm{e}-4)-$ \\
\hline
\end{tabular}
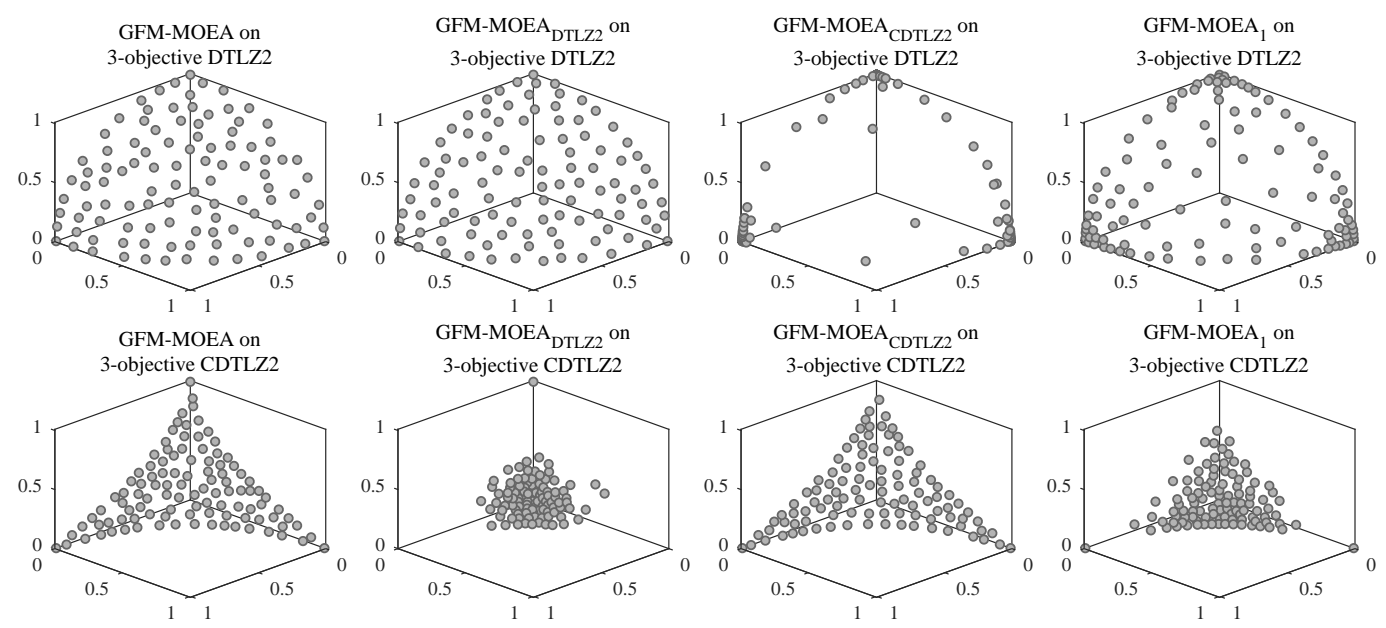

Fig. 4. The non-dominated solution set with the median HV among 30 runs obtained by GFM-MOEA and its three variants on DTLZ2 and CDTLZ2 with 3 objectives.

identical with the true PF of DTLZ2, GFM-MOEA ${ }_{D T L Z 2}$ can obtain similar HV values with the proposed GFMMOEA on DTLZ2. However, its performance considerably deteriorates when meeting CDTLZ2, since the model is concave but the true PF of CDTLZ2 is convex. Due to the same reason, the performance of GFMMOEA $_{C D T L Z 2}$ is as good as GFM-MOEA on CDTLZ2, but GFM-MOEA ${ }_{C D T L Z 2}$ obtains a non-dominated solution set with poor diversity on DTLZ2. Thirdly, for GFM$\mathrm{MOEA}_{1}$ which adopts a linear front model, its performance is not satisfactory on either DTLZ2 or CDTLZ2. This is mainly due to the fact that the linear front model is identical with neither DTLZ2 nor CDTLZ2.

In summary, the proposed GFM can adaptively estimate the PF models of different geometries, such that GFM-MOEA performs consistently in approximating the PFs of both DTLZ2 and CDTLZ2. By contrast, a constant model setting cannot make GFM-MOEA work well on MOPs with various PFs. This confirms that the proposed GFM is crucial to the performance of GFM-MOEA.

In order to further assess the accuracy of the proposed GFM method during the optimization procedure, we record the MSE values returned by GFM with respect to 10000 points sampled on the true PF of 3-objective DTLZ1, DTLZ2, DTLZ5, DTLZ7, CDTLZ2, and IDTLZ2, averaging over 30 runs. As shown in Fig. 5, the MSE values are gradually decreased as the number of generations increase, which indicates promising convergence

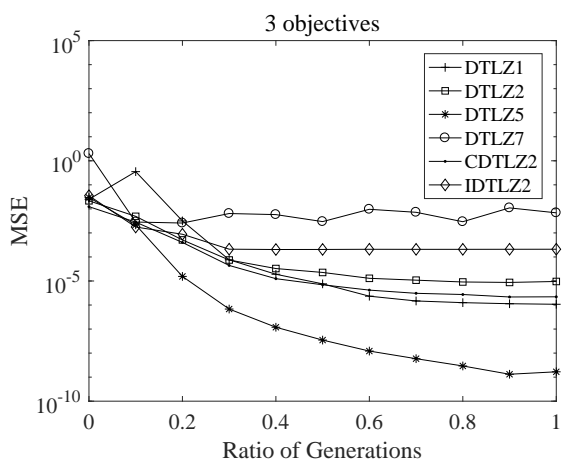

Fig. 5. MSE values returned by GFM during the optimization procedure of GFM-MOEA on 3-objective DTLZ1, DTLZ2, DTLZ5, DTLZ7, CDTLZ2, and IDTLZ2, with respect to 10000 points sampled on each true $\mathrm{PF}$, averaging over 30 runs.

of GFM. In addition, as shown in Fig. 6, the similarity between the estimated PF and the true PF of DTLZ1 is also substantially increased with respect to the number of generations. Such observations have further confirmed the accuracy of the proposed GFM method.

\section{Performance on MOPs}

The experimental results of GFM-MOEA, NSGA-II, MOEA/D, IBEA, and MOEA/D-AWA on 2-objective ZDT1-ZDT4 and ZDT6, and 3-objective DTLZ1-DTLZ7, CDTLZ2, IDTLZ1, IDTLZ2, WFG1-WFG9, and IWFG1IWFG4 are listed in Table III. According to the statisti- 
TABLE III

Statistical Results of IGD VAlues Obtained by NSGA-II, MOEA/D, IBEA, MOEA/D-AWA, AND GFM-MOEA ON 2-ObJeCtive ZDT1-ZDT4 AND ZDT6, AND 3-OBJECTIVE DTLZ1-DTLZ7, CDTLZ2, IDTLZ1, IDTLZ2, WFG1-WFG9, AND IWFG1-IWFG4.

\begin{tabular}{|c|c|c|c|c|c|c|}
\hline Problem & $M$ & NSGA-II & MOEA/D & IBEA & MOEA/D-AWA & GFM-MOEA \\
\hline & \multirow{5}{*}{2} & )$\approx$ & & 2) - & & \\
\hline ZDT2 & & $2 e-2$ & & $e-2$ & 2) - & \\
\hline ZDT3 & & $291 \mathrm{e}-2(8.26 \mathrm{e}-2) \approx$ & $927 e-2(3.08 e-2)+$ & $943 e-1(9.27 e-2)-$ & $6284 \mathrm{e}-2(3.03 \mathrm{e}-2)+$ & 4604 \\
\hline ZDT4 & & $995 \mathrm{e}-3(4.26 \mathrm{e}-3)-$ & $1.5782 \mathrm{e}-2(5.32 \mathrm{e}-3)-$ & $8472 \mathrm{e}-1(1.20 \mathrm{e}-1)-$ & $2.6845 \mathrm{e}-2(2.59 \mathrm{e}-2)-$ & $.7450 e-3(3.63 e-3)$ \\
\hline ZDT6 & & $7486 \mathrm{e}-3(1.14 \mathrm{e}-3)-$ & $3.7408 \mathrm{e}-3(3.98 \mathrm{e}-4)-$ & $5.1791 \mathrm{e}-3(2.68 \mathrm{e}-4)-$ & $3.6268 \mathrm{e}-3(6.58 \mathrm{e}-4) \approx$ & $.5422 \mathrm{e}-3$ \\
\hline DTLZ1 & & $2.6605 \mathrm{e}-2(1.50 \mathrm{e}-3)-$ & $1.9048 \mathrm{e}-2(7.81 \mathrm{e}-5)+$ & $1.5801 \mathrm{e}-1(3.24 \mathrm{e}-2)-$ & $2.2609 \mathrm{e}-2(4.95 \mathrm{e}-3) \approx$ & $1.9665 \mathrm{e}-2(1.48 \mathrm{e}-4)$ \\
\hline DTLZ2 & & $5 e-2(2.65 e-3)-$ & $5.1592 \mathrm{e}-2(4.67 \mathrm{e}-4)+$ & $7.8361 \mathrm{e}-2(2.51$ & 5.140 & 5.199 \\
\hline DTLZ3 & & $5 e-2(7.59 e-3)-$ & $5.4274 \mathrm{e}-2$ & $4.7767 \mathrm{e}-1(9.18$ & 5.283 & $28 e-3)$ \\
\hline DTLZ4 & & $4 \mathrm{e}-1(2.69 \mathrm{e}-1)-$ & $4.0802 \mathrm{e}-1(2.70 \mathrm{e}-1)-$ & $7 e-2(2.52 e$ & $-1) \approx$ & \\
\hline DTLZ5 & & $8 e-3(2.86 e-4)-$ & $1.8542 \mathrm{e}-2$ & $1.5397 \mathrm{e}-2(1.66$ & $-4)-$ & \\
\hline DTLZ6 & & 3e-3 & $23 e-2$ & e-2 ( & 4) - & \\
\hline DTLZ7 & & - & $6 e-1$ & 3) - & 1) - & \\
\hline CDTLZ2 & & 3) - & 3) - & 3) - & 3.72 & \\
\hline ID & & & & 3) - & & \\
\hline IDTLZ2 & & 3) - & $0 e-2$ & $-2)-$ & -3) - & 5.50 \\
\hline WFG1 & \multirow{13}{*}{3} & $2.4734 \mathrm{e}-1(2.45 \mathrm{e}-2)-$ & $2.3377 \mathrm{e}-1(1.34 \mathrm{e}-2)-$ & $2.0288 \mathrm{e}-1(9.43 \mathrm{e}-3)-$ & $4.3645 \mathrm{e}-1(8.39 \mathrm{e}-2)-$ & $1.7105 \mathrm{e}-1$ \\
\hline WFG2 & & $0 e-3)-$ & $4.1286 \mathrm{e}-1$ & $2.5243 \mathrm{e}-1$ & 1.71 & 3e-3) \\
\hline WFG3 & & -2) - & $1.1947 \mathrm{e}-1$ & -3) + & $-2) \approx$ & 40e-3) \\
\hline WFG4 & & $7 e-3)-$ & $2.8132 \mathrm{e}-1$ & $0 e-1(1.27 e-2)-$ & $2.0477 \mathrm{e}-1$ & 2.0503 \\
\hline WFG5 & & $(e-2)-$ & $2.7816 \mathrm{e}-1$ & e-2) - & e-3) $\approx$ & $9 e-3)$ \\
\hline G6 & & -2) - & 2) - & 2) - & 2) - & $e-2)$ \\
\hline FG7 & & 2) - & 3) - & 2) - & 2.052 & 2050 \\
\hline G8 & & 2) - & 3) - & 2) - & 2.91 & 2.74 \\
\hline G99 & & $e-2)-$ & e-1 & 3) - & 2.32 & $e-3)$ \\
\hline IV & & $\approx$ & ) & 1) + & 1) $\approx$ & 5.19 \\
\hline IWFG2 & & $80 \mathrm{e}-1(1.99 \mathrm{e}-2)-$ & $5.2741 \mathrm{e}-1(3.87 \mathrm{e}-2)-$ & $3.5802 \mathrm{e}-1(1.53 \mathrm{e}-2)-$ & $3.4549 \mathrm{e}-1(1.94 \mathrm{e}-2)-$ & 3.120 \\
\hline IWFG3 & & $8.3841 \mathrm{e}-2(9.21 \mathrm{e}-3)+$ & $1.4417 e-1(9.95 e-3)-$ & $4.2887 \mathrm{e}-2(2.69 \mathrm{e}-3)+$ & $7.4535 \mathrm{e}-2$ & 1.091 \\
\hline IWFG4 & & $2.6623 \mathrm{e}-1(1.11 \mathrm{e}-2)-$ & $4.5557 \mathrm{e}-1(4.22 \mathrm{e}-3)-$ & $3.1920 \mathrm{e}-1(2.17 \mathrm{e}-2)-$ & $2.0776 \mathrm{e}-1(2.80 \mathrm{e}-3)+$ & $2.1584 \mathrm{e}-1(3.51 \mathrm{e}-3)$ \\
\hline$+1-1$ & & $1 / 24 / 3$ & $3 / 25 / 0$ & $4 / 24 / 0$ & $4 / 14 / 10$ & \\
\hline
\end{tabular}

cal results in terms of IGD metric, NSGA-II performs the best on ZDT1, MOEA/D performs the best on ZDT3 and DTLZ1, IBEA outperforms the other compared algorithms on DTLZ4, WFG3, IWFG1, and IWFG3, MOEA/D-AWA performs better than the compared algorithms on DTLZ2, DTLZ3, WFG4, WFG7, and IWFG4, and GFM-MOEA gains the best results on all the remaining 16 MOPs. In conclusion, GFM-MOEA is competitive to the four compared MOEAs on MOPs with simple PFs (ZDT1, ZDT2, ZDT4, ZDT6, DTLZ1-DTLZ4, and CDTLZ2), while it performs remarkably better than them on MOPs with complex PFs (DTLZ5-DTLZ7, IDTLZ1, and IDTLZ2) and scaled PFs (WFG1, WFG2, WFG5, WFG6, WFG8, WFG9, and IWFG2).

Fig. 7 shows the non-dominated solution set with the median IGD obtained by the five compared MOEAs on DTLZ2, CDTLZ2, and IWFG4. For NSGA-II, it first sorts the solutions based on their Pareto dominance relations, and then distinguishes the solutions in the same front by crowding distance. However, it seems that the crowding distance is not very effective in diversity preservation of NSGA-II on MOPs, since the distribution of the solutions obtained by NSGA-II is satisfactory on none of the three MOPs as shown in Fig. 7.

As for MOEA/D, it makes each solution converge to the same direction with one of the predefined uniformly distributed weight vectors, thus the final population can hold the same uniformity with the weight vectors. As shown in the second column of Fig. 7, the solutions obtained by MOEA/D have a good distribution on DTLZ2, except that the solutions located on the border are slightly more crowded than those located in the middle. This should be attributed to the reason mentioned in Section I, where the weight vectors are uniformly distributed on a hyperplane, hence the solutions on the border will be more crowded than those in the middle when they are mapped to a concave surface. This phenomenon becomes worse on CDTLZ2, where almost all the solutions obtained by MOEA/D are located in the middle of the PF. For IWFG4, however, since the predefined weight vectors can only cover part of the inverted PF, MOEA/D is unable to make all the solutions distribute uniformly on the PF.

IBEA is an indicator based MOEA, where the selection criterion is defined on the basis of a binary indicator. According to the solutions obtained by IBEA shown in Fig. 7, the indicator employed by IBEA is easily biased, which makes the solutions distributed in the middle of the PFs of DTLZ2, CDTLZ2 and IWFG4. Regarding the variant of MOEA/D tailored for solving MOPs with complex PFs, MOEA/D-AWA, it obtains an obviously better distribution of solutions than MOEA/D on MOPs with complex PFs. In contrast to the above four MOEAs, by measuring the solutions according to the PF estimated by GFM, the solutions obtained by GFM-MOEA distribute well on all of the three MOPs, no matter whether the true PF is concave, convex or inverted. Therefore, GFM-MOEA has better performance 

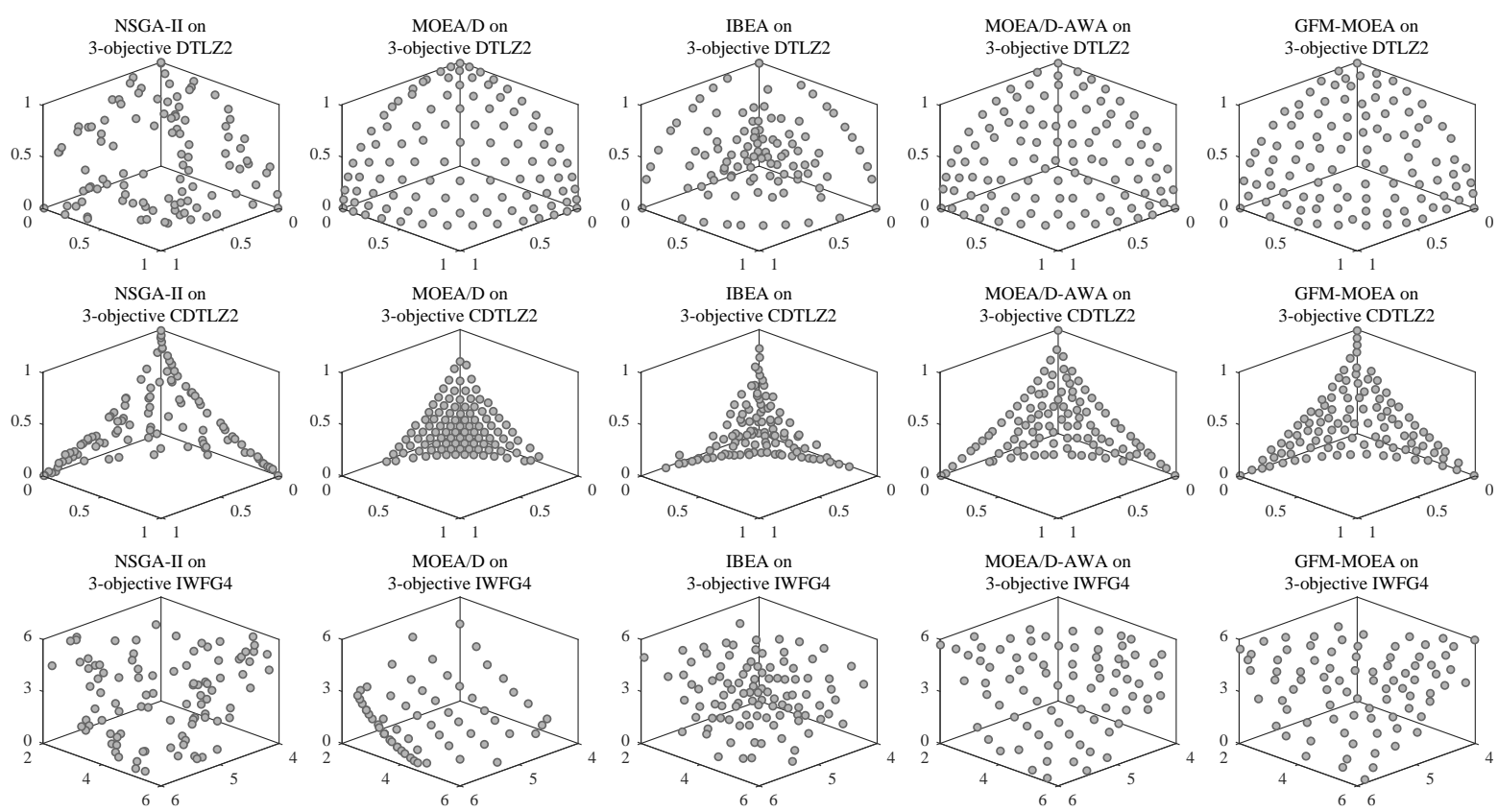

Fig. 7. The non-dominated solution set with the median IGD among 30 runs obtained by NSGA-II, MOEA/D, IBEA, MOEA/D-AWA, and GFM-MOEA on DTLZ2, CDTLZ2, and IDTLZ2 with 3 objectives.
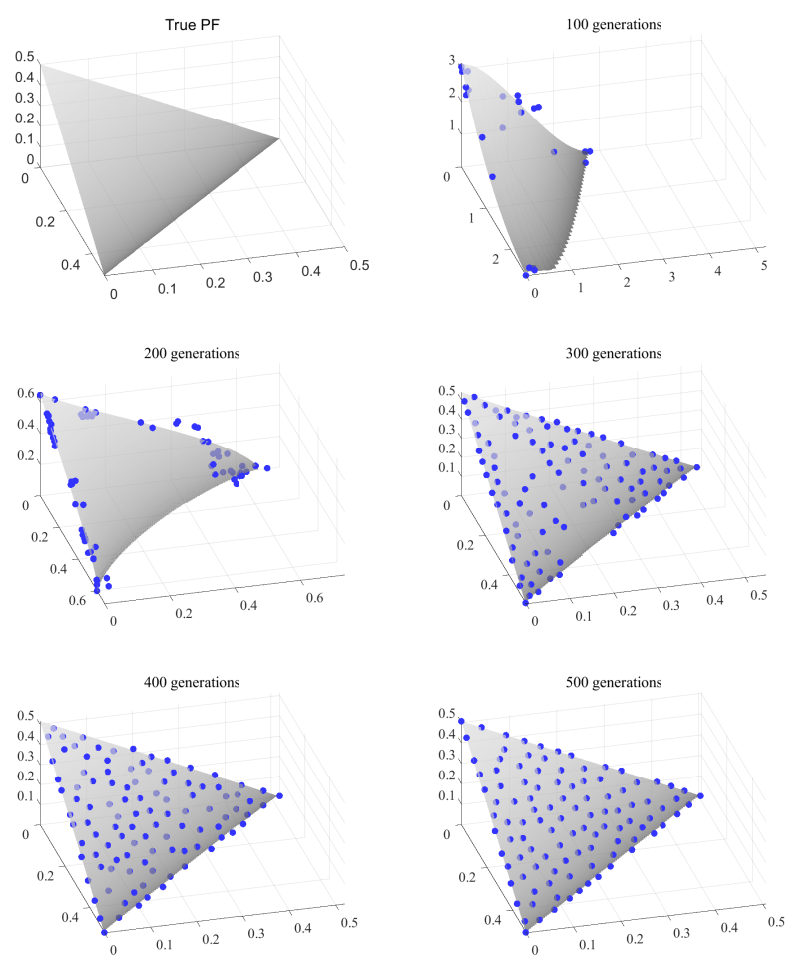

Fig. 6. Estimated PFs obtained at different generations of GFM-MOEA on 3-objective DLTZ1, where the non-dominated solutions are shown in solid points, and the estimated PFs are shown in surfaces.

consistency in solving MOPs with different types of PFs in comparison with existing MOEAs.

\section{Perfromance on $\mathrm{MaOPs}$}

In this subsection, the proposed GFM-MOEA is compared with MOEA/DD, RVEA, MOEA/D-PaS, and
VaEA for solving MaOPs. The HV values of the four compared MOEAs on 10-objective DTLZ and WFG problems are listed in Table IV. As shown in the table, GFMMOEA performs the best on 16 of the 23 test instances in terms of $\mathrm{HV}$, while the numbers of best results obtained by MOEA/DD, RVEA, MOEA/D-PaS, and VaEA are 2, 3,1 and 1, respectively. In terms of the Wilcoxon rank sum test, the proportion of test instances where GFMMOEA performs significantly better than MOEA/DD, RVEA, MOEA/D-PaS, and VaEA is 16/23, 17/23, 21/23 and $20 / 23$, respectively.

For visual observations of the differences between the results, Fig. 8 plots the non-dominated solution sets with the median $\mathrm{HV}$ values among 30 runs obtained by the five MOEAs on WFG1 and IWFG1. It is clear that VaEA and GFM-MOEA can find a set of solutions covering the whole PF of IWFG1, while only GFM-MOEA can exhibit a good diversity performance on WFG1. As a result, GFM-MOEA is also able to perform consistently on MaOPs with different types of PFs.

The statistical results of MOEA/DD, RVEA, MOEA/D-PaS, VaEA, and GFM-MOEA on 2-objective ZDT problems and 3-objective DTLZ and WFG problems can be found in Supplementary Materials I. In order to verify the performance of these MOEAs on more challenging MaOPs, they are tested on MaF1-MaF15, which are designed for IEEE CEC'2018 Competition on Evolutionary Many-Objective Optimization and contain diverse and complicated PFs. The corresponding results can be found in Supplementary Materials II. The non-dominated solution set with median performance obtained by all the 9 compared MOEAs on all the test instances can be found in Supplementary Materials 
TABLE IV

Statistical Results of HV VAlues Obtained by MOEA/DD, RVEA, MOEA/D-PAS, VAEA, AND GFM-MOEA ON DTLZ1-DTLZ7, CDTLZ2, IDTLZ1, IDTLZ2, WFG1-WFG9, AND IWFG1-IWFG4 WITH 10 OBJECTIVES.

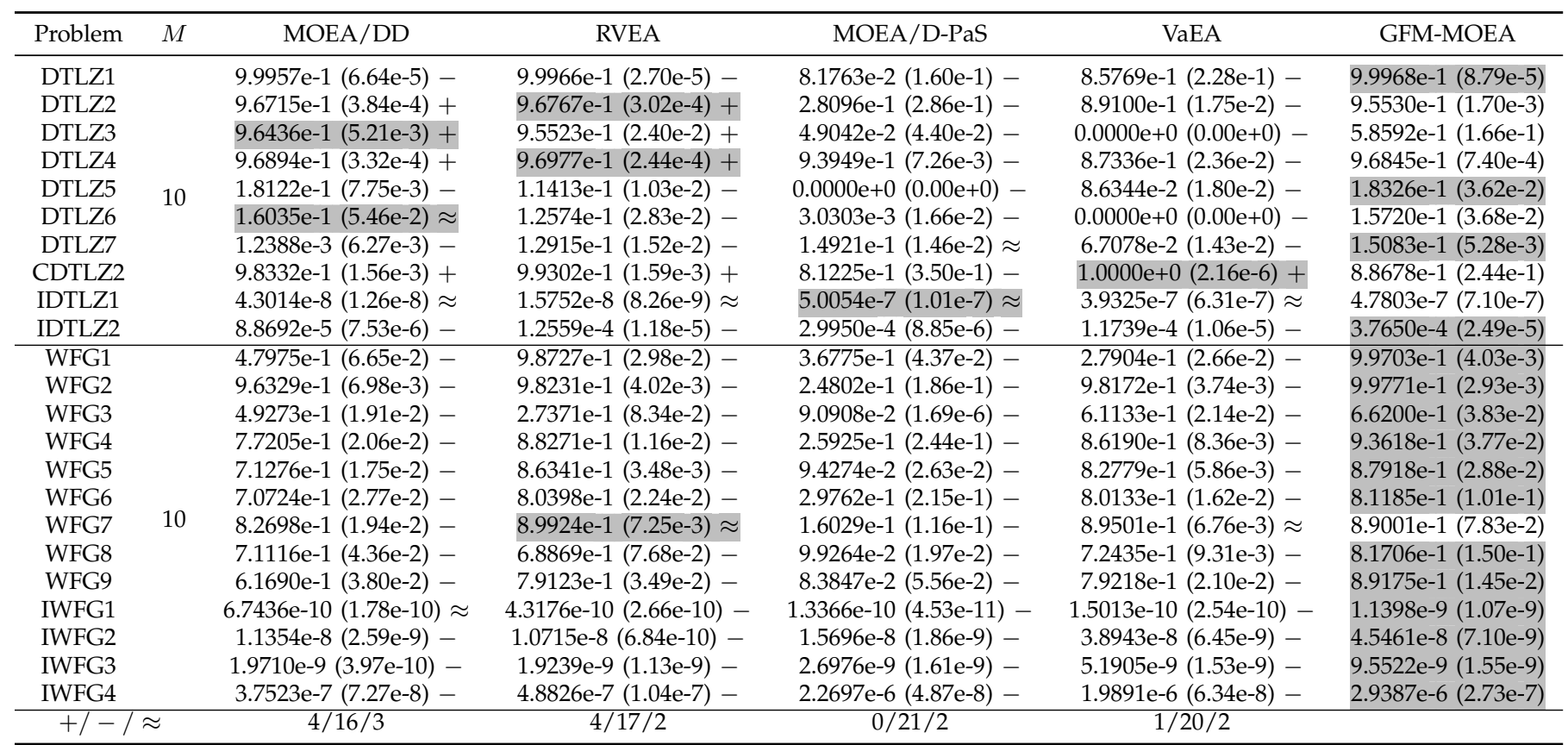
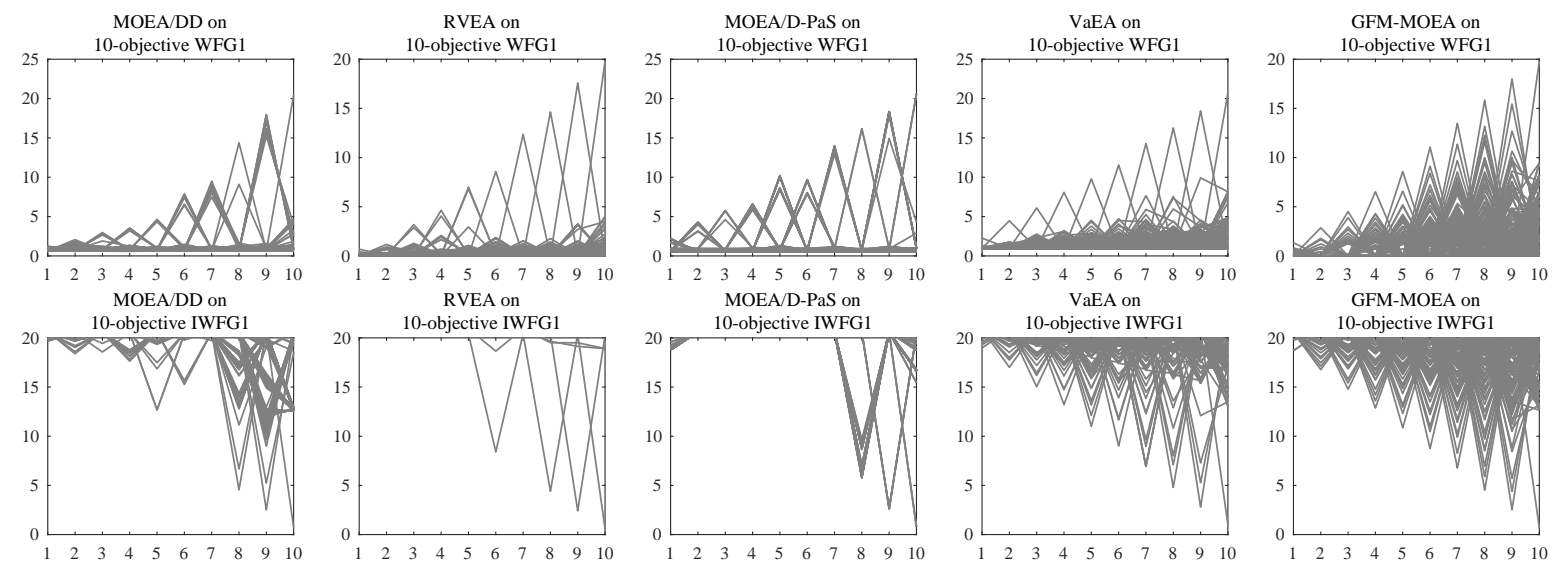

Fig. 8. The non-dominated solution set with the median HV among 30 runs obtained by MOEA/DD, RVEA, MOEA/D-PaS, VaEA, and GFM-MOEA on WFG1 and IWFG1 with 10 objectives.

III. Furthermore, the comparison between GFM-MOEA and more state-of-the-art MOEAs can be found in Supplementary Materials IV.

\section{E. Parameter Sensitivity Analysis}

There are two parameters which need to be set in GFM-MOEA, i.e., the penalty parameter $\theta$ and the frequency $f_{r}$. Here, we investigate the influence of the two parameters on the performance of GFM-MOEA.

Fig. 9 shows the average HV values of GFM-MOEA on DTLZ1, DTLZ2, DTLZ5, DTLZ7, CDTLZ2, and IDTLZ2 with 3 and 10 objectives over 30 runs, where the parameter $\theta$ is varied from 0 to 1 and $f_{r}$ is fixed to 0.1 . It can be seen from the figure that the HV value rapidly decreases when $\theta$ varies from 0.4 to 1 , and it increases on MOPs with 10 objectives when $\theta$ varies from 0 to
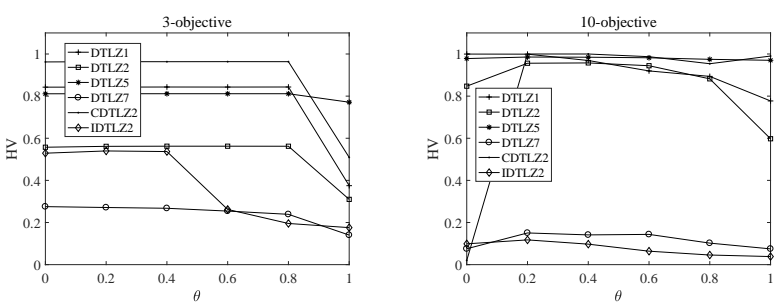

Fig. 9. The average HV values of GFM-MOEA on DTLZ1, DTLZ2, DTLZ5, DTLZ7, CDTLZ2, and IDTLZ2 with 3 and 10 objectives over 30 runs, where $\theta$ is varied from 0 to 1 and $f_{r}$ is fixed to 0.1 .

0.2 . As a result, $\theta=0.2$ is recommended for consistent performance.

Fig. 10 shows the average HV values of GFM-MOEA on the same test instances, where the parameter $f_{r}$ is 

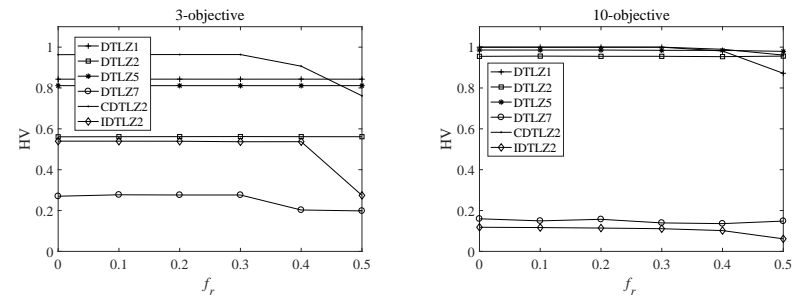

Fig. 10. The average HV values of GFM-MOEA on DTLZ1, DTLZ2, DTLZ5, DTLZ7, CDTLZ2, and IDTLZ2 with 3 and 10 objectives over 30 runs, where $\theta$ is fixed to 0.2 and $f_{r}$ is varied from 0 to 0.5 .

varied from 0 to 0.5 and $\theta$ is fixed to 0.2 . Note that $f_{r}=0$ indicates that GFM is performed at each generation, and $f_{r}=0.5$ implies that GFM is performed only once in one run. It turns out that the HV value decreases on some test instances when $f_{r}$ is larger than 0.3. Therefore, $f_{r}$ needs to be set to a relatively small value. By considering the balance between efficiency and effectiveness, $f_{r}=0.1$ is suggested in all cases.

In summary, it can be concluded from the above observations that the performance of GFM-MOEA is insensitive to the settings of $\theta$ and $f_{r}$, as long as that $f_{r}$ is smaller than 0.3 and $\theta$ is between 0.2 and 0.4 .

\section{F. Influence of the Ideal Point}

As mentioned in Section III-A, in order to make the PF of the MOP to be solved consistent with the model in GFM, the objective values are translated according to the current ideal point before training the model, i.e., $f_{i}^{\prime}=$ $f_{i}-z_{i}^{*}$. However, some recent studies [17], [34] pointed out that a point lower than the current ideal point can lead to better performance, i.e., $f_{i}^{\prime}=f_{i}-\left(z_{i}^{*}-\epsilon_{i}\right)$ with $\epsilon_{i}>0$. To investigate the effect of the usage of the current ideal point, we follow the settings in [34] to compare GFM-MOEA with two of its variants GFM-MOEA* and GFM-MOEA**, where $\epsilon_{i}$ is set to 1 and linearly varied from 1 to 0 during the optimization process, respectively.

Table $\mathrm{V}$ lists the average $\mathrm{HV}$ values obtained by the three MOEAs on WFG1-WFG9 with 3 and 10 objectives over 30 runs. The statistical results show the superiority of the original GFM-MOEA over the two variants, hence it can be confirmed that the original current ideal point (i.e., $\epsilon_{i}=0$ ) is the best for GFM-MOEA. This is due to the fact that a point lower than the current ideal point can increase the estimation error of GFM, thus leading to a poor performance of the proposed GFM-MOEA. As evidenced by the results in Table VI, the MSE values returned by GFM in the final generation of GFM-MOEA are significantly smaller than those in GFM-MOEA* and GFM-MOEA** on most test instances.

\section{CONCLUSIONS}

In this paper, we have proposed a generic front modeling (GFM) method for enhancing the performance consistency of MOEAs in tackling MOPs and MaOPs with various PFs. By using the non-dominated solutions
TABLE $\mathrm{V}$

Statistical Results of HV VAlues Obtained by GFM-MOEA $(\epsilon=0)$, GFM-MOEA* $(\epsilon=1)$, AND GFM-MOEA ${ }^{* *}(\epsilon$ VARIES FROM 1 TO 0) ON WFG1-WFG9 WITH 3 AND 10 OBJECTIVES.

\begin{tabular}{cccc}
\hline Problem $(M)$ & GFM-MOEA & GFM-MOEA* & GFM-MOEA** \\
\hline WFG1(3) & $9.4264 \mathrm{e}-1$ & $9.4485 \mathrm{e}-1+$ & $8.8631 \mathrm{e}-1-$ \\
WFG2(3) & $9.3859 \mathrm{e}-1$ & $9.3657 \mathrm{e}-1-$ & $8.6673 \mathrm{e}-1-$ \\
WFG3(3) & $6.2471 \mathrm{e}-1$ & $6.3173 \mathrm{e}-1+$ & $6.2749 \mathrm{e}-1 \approx$ \\
WFG4(3) & $5.5403 \mathrm{e}-1$ & $5.2736 \mathrm{e}-1-$ & $5.5418 \mathrm{e}-1 \approx$ \\
WFG5(3) & $5.1705 \mathrm{e}-1$ & $4.9338 \mathrm{e}-1-$ & $5.1929 \mathrm{e}-1 \approx$ \\
WFG6(3) & $5.0347 \mathrm{e}-1$ & $4.7944 \mathrm{e}-1-$ & $5.0728 \mathrm{e}-1 \approx$ \\
WFG7(3) & $5.5820 \mathrm{e}-1$ & $5.3197 \mathrm{e}-1-$ & $5.5905 \mathrm{e}-1 \approx$ \\
WFG8(3) & $4.7208 \mathrm{e}-1$ & $4.4407 \mathrm{e}-1-$ & $4.5295 \mathrm{e}-1-$ \\
WFG9(3) & $5.3062 \mathrm{e}-1$ & $5.1716 \mathrm{e}-1-$ & $5.3575 \mathrm{e}-1+$ \\
\hline WFG1(10) & $9.9302 \mathrm{e}-1$ & $9.2575 \mathrm{e}-1-$ & $9.2892 \mathrm{e}-1 \approx$ \\
WFG2(10) & $9.9940 \mathrm{e}-1$ & $9.9633 \mathrm{e}-1-$ & $9.9775 \mathrm{e}-1-$ \\
WFG3(10) & $6.5881 \mathrm{e}-1$ & $3.0158 \mathrm{e}-1-$ & $6.1037 \mathrm{e}-1-$ \\
WFG4(10) & $9.4462 \mathrm{e}-1$ & $7.0604 \mathrm{e}-1-$ & $8.8783 \mathrm{e}-1-$ \\
WFG5(10) & $8.8371 \mathrm{e}-1$ & $6.6122 \mathrm{e}-1-$ & $8.2751 \mathrm{e}-1-$ \\
WFG6(10) & $8.4153 \mathrm{e}-1$ & $5.2082 \mathrm{e}-1-$ & $7.7197 \mathrm{e}-1-$ \\
WFG7(10) & $9.5153 \mathrm{e}-1$ & $7.0364 \mathrm{e}-1-$ & $8.9591 \mathrm{e}-1-$ \\
WFG8(10) & $8.8017 \mathrm{e}-1$ & $4.7723 \mathrm{e}-1-$ & $7.8186 \mathrm{e}-1-$ \\
WFG9(10) & $8.9458 \mathrm{e}-1$ & $6.5686 \mathrm{e}-1-$ & $8.6408 \mathrm{e}-1-$ \\
\hline$+/-/ \approx$ & & $2 / 16 / 0$ & $1 / 11 / 6$ \\
\hline
\end{tabular}

TABLE VI

MSE VALUES RETURNED BY GFM IN THE FINAL GENERATION OF GFM-MOEA $(\epsilon=0)$, GFM-MOEA* $(\epsilon=1)$, AND GFM-MOEA** $(\epsilon$ VARIES FROM 1 TO 0) ON WFG1-WFG9 WITH 3 AND 10 OBJECTIVES.

\begin{tabular}{cccc}
\hline Problem $(M)$ & GFM-MOEA & GFM-MOEA* & GFM-MOEA** \\
\hline WFG1(3) & $5.9894 \mathrm{e}-4$ & $1.6484 \mathrm{e}-2$ & $1.1754 \mathrm{e}-3$ \\
WFG2(3) & $2.7157 \mathrm{e}-3$ & $1.2361 \mathrm{e}-1$ & $3.6594 \mathrm{e}-3$ \\
WFG3(3) & $4.7587 \mathrm{e}-4$ & $3.0309 \mathrm{e}-2$ & $4.4592 \mathrm{e}-4$ \\
WFG4(3) & $2.4633 \mathrm{e}-5$ & $6.2632 \mathrm{e}-3$ & $9.7221 \mathrm{e}-5$ \\
WFG5(3) & $2.4426 \mathrm{e}-6$ & $2.2003 \mathrm{e}-3$ & $3.5608 \mathrm{e}-5$ \\
WFG6(3) & $1.5297 \mathrm{e}-5$ & $6.7826 \mathrm{e}-3$ & $6.6286 \mathrm{e}-5$ \\
WFG7(3) & $8.1687 \mathrm{e}-6$ & $6.1851 \mathrm{e}-3$ & $5.9037 \mathrm{e}-5$ \\
WFG8(3) & $9.4101 \mathrm{e}-3$ & $5.9348 \mathrm{e}-3$ & $1.2672 \mathrm{e}-2$ \\
WFG9(3) & $1.2351 \mathrm{e}-5$ & $6.2160 \mathrm{e}-3$ & $4.9766 \mathrm{e}-5$ \\
\hline WFG1(10) & $3.5386 \mathrm{e}-3$ & $3.4497 \mathrm{e}-1$ & $1.9321 \mathrm{e}+0$ \\
WFG2(10) & $1.9491 \mathrm{e}-1$ & $3.4885 \mathrm{e}-1$ & $3.3299 \mathrm{e}-1$ \\
WFG3(10) & $4.5379 \mathrm{e}-1$ & $1.3974 \mathrm{e}+0$ & $1.4801 \mathrm{e}-1$ \\
WFG4(10) & $8.0666 \mathrm{e}-2$ & $8.0176 \mathrm{e}-1$ & $3.4410 \mathrm{e}-1$ \\
WFG5(10) & $5.5038 \mathrm{e}-2$ & $8.6427 \mathrm{e}-1$ & $4.1027 \mathrm{e}-1$ \\
WFG6(10) & $8.2024 \mathrm{e}-2$ & $1.3527 \mathrm{e}+0$ & $4.2289 \mathrm{e}+0$ \\
WFG7(10) & $8.4648 \mathrm{e}-2$ & $9.4211 \mathrm{e}-1$ & $5.0458 \mathrm{e}-1$ \\
WFG8(10) & $3.6861 \mathrm{e}-2$ & $1.1035 \mathrm{e}+0$ & $1.2342 \mathrm{e}-1$ \\
WFG9(10) & $5.4451 \mathrm{e}-2$ & $8.0844 \mathrm{e}-1$ & $2.1439 \mathrm{e}-1$ \\
\hline
\end{tabular}

in the population as training data, the proposed GFM adopts the Levenberg-Marquardt algorithm to iteratively model the non-dominated front of the problem to be solved. The empirical results have demonstrated that GFM is capable of modeling fronts of various shapes with low estimation errors.

Additionally, an MOEA has been developed by incorporating the proposed GFM in the calculation of fitness value. With the assistance of the fronts estimated by GFM, the proposed MOEAs can measure the fitness of the candidate solutions in a simple manner, and the performance consistency of the MOEA in tackling MOPs (as well as MaOPs) with various PFs is significantly enhanced. As evidenced by the experiment results, the proposed GFM-MOEA performs consistently on a variety of benchmark test problems, and its performance is 
superior over several state-of-the-art MOEAs on most of them.

In the future, we would like to further investigate the following two issues. First, since the proposed GFM is based on a simplex-like model, its performance may deteriorate if the $\mathrm{PF}$ is substantially different from a simplex-like shape (e.g., VNT3 [51]), so it is interesting to further investigate how to estimate such special PFs. Second, we would like to embed GFM into decomposition or indicator based MOEAs to see if it is able to enhance their performance as well. In addition, it is also interesting to investigate the scalability of GFM on largescale MOPs and MaOPs [52].

\section{REFERENCES}

[1] S. H. Yeung, K. F. Man, K. M. Luk, and C. H. Chan, "A trapeizform U-slot folded patch feed antenna design optimized with jumping genes evolutionary algorithm," IEEE Transactions on Antennas and Propagation, vol. 56, no. 2, pp. 571-577, 2008.

[2] J. G. Herrero, A. Berlanga, and J. M. M. López, "Effective evolutionary algorithms for many-specifications attainment: Application to air traffic control tracking filters," IEEE Transactions on Evolutionary Computation, vol. 13, no. 1, pp. 151-168, 2009.

[3] R. C. Purshouse and P. J. Fleming, "On the evolutionary optimization of many conflicting objectives," IEEE Transactions on Evolutionary Computation, vol. 11, no. 6, pp. 770-784, 2007.

[4] A. Zhou, B.-Y. Qu, H. Li, S.-Z. Zhao, P. N. Suganthan, and Q. Zhang, "Multiobjective evolutionary algorithms: A survey of the state of the art," Swarm and Evolutionary Computation, vol. 1, no. 1, pp. 32-49, 2011.

[5] X. Zhang, Y. Tian, R. Cheng, and Y. Jin, "An efficient approach to non-dominated sorting for evolutionary multi-objective optimization," IEEE Transactions on Evolutionary Computation, vol. 19, no. 2, pp. 201-213, 2015.

[6] K. Deb, A. Pratap, S. Agarwal, and T. Meyarivan, "A fast and elitist multi-objective genetic algorithm: NSGA-II," IEEE Transactions on Evolutionary Computation, vol. 6, no. 2, pp. 182-197, 2002.

[7] C. He, Y. Tian, Y. Jin, X. Zhang, and L. Pan, "A radial space division based evolutionary algorithm for many-objective optimization," Applied Soft Computing, vol. 61, pp. 603-621, 2017.

[8] Q. Zhang and H. Li, "MOEA/D: A multi-objective evolutionary algorithm based on decomposition," IEEE Transactions on Evolutionary Computation, vol. 11, no. 6, pp. 712-731, 2007.

[9] K. Deb and H. Jain, "An evolutionary many-objective optimization algorithm using reference-point based non-dominated sorting approach, part I: Solving problems with box constraints," IEEE Transactions on Evolutionary Computation, vol. 18, no. 4, pp. 577-601, 2014.

[10] L. While, P. Hingston, L. Barone, and S. Huband, "A faster algorithm for calculating hypervolume," IEEE Transactions on Evolutionary Computation, vol. 10, no. 1, pp. 29-38, 2006.

[11] A. Zhou, Y. Jin, Q. Zhang, B. Sendhoff, and E. Tsang, "Combining model-based and genetics-based offspring generation for multiobjective optimization using a convergence criterion," in Proceedings of the 2006 IEEE Congress on Evolutionary Computation, 2006, pp. 892-899.

[12] H. Trautmann, T. Wagner, and D. Brockhoff, "R2-EMOA: Focused multiobjective search using R2-indicator-based selection," in Proceedings of the 7th International Conference on Learning and Intelligent Optimization, 2013, pp. 70-74.

[13] J. Bader and E. Zitzler, "HypE: An algorithm for fast hypervolume-based many-objective optimization," Evolutionary Computation, vol. 19, no. 1, pp. 45-76, 2011.

[14] R. Hernández Gómez and C. A. Coello Coello, "Improved metaheuristic based on the R2 indicator for many-objective optimization," in Proceedings of the 2015 on Genetic and Evolutionary Computation Conference, 2015, pp. 679-686.

[15] H. Ishibuchi, Y. Setoguchi, H. Masuda, and Y. Nojima, "Performance of decomposition-based many-objective algorithms strongly depends on Pareto front shapes," IEEE Transactions on Evolutionary Computation, vol. 21, no. 2, pp. 169-190, 2017.
[16] R. Cheng, M. Li, Y. Tian, X. Zhang, S. Yang, Y. Jin, and X. Yao, “A benchmark test suite for evolutionary many-objective optimization," Complex \& Intelligent Systems, vol. 3, no. 1, pp. 67-81, 2017.

[17] Y. Qi, X. Ma, F. Liu, L. Jiao, J. Sun, and J. Wu, "MOEA/D with adaptive weight adjustment," Evolutionary computation, vol. 22, no. 2, pp. 231-264, 2014.

[18] N. Beume, B. Naujoks, and M. Emmerich, "SMS-EMOA: Multiobjective selection based on dominated hypervolume," European Journal of Operational Research, vol. 181, no. 3, pp. 1653-1669, 2007.

[19] X. Zhang, Y. Tian, and Y. Jin, "A knee point driven evolutionary algorithm for many-objective optimization," IEEE Transactions on Evolutionary Computation, vol. 19, no. 6, pp. 761-776, 2015.

[20] R. Wang, Q. Zhang, and T. Zhang, "Decomposition based algorithms using Pareto adaptive scalarizing methods," IEEE Transactions on Evolutionary Computation, vol. 20, no. 6, pp. 821-837, 2016.

[21] R. Cheng, Y. Jin, M. Olhofer, and B. Sendhoff, "A reference vector guided evolutionary algorithm for many-objective optimization," IEEE Transactions on Evolutionary Computation, vol. 20, no. 5, pp. 773-791, 2016.

[22] Y. Jin and B. Sendhoff, "Connectedness, regularity and the success of local search in evolutionary multi-objective optimization," in Proceedings of the 2003 IEEE Congress on Evolutionary Computation, vol. 3, 2003, pp. 1910-1917.

[23] Y. Liu, D. Gong, J. Sun, and Y. Jin, "A many-objective evolutionary algorithm using a one-by-one selection strategy," IEEE Transactions on Cybernetics, vol. 47, no. 9, pp. 2689-2702, 2017.

[24] H. Jain and K. Deb, "An evolutionary many-objective optimization algorithm using reference-point based nondominated sorting approach, part II: Handling constraints and extending to an adaptive approach," IEEE Transactions on Evolutionary Computation, vol. 18, no. 4, pp. 602-622, 2014.

[25] Y. Tian, R. Cheng, X. Zhang, F. Cheng, and Y. Jin, "An indicator based multi-objective evolutionary algorithm with reference point adaptation for better versatility," IEEE Transactions on Evolutionary Computation, 2017, in press.

[26] H. Ishibuchi, K. Doi, and Y. Nojima, "On the effect of normalization in MOEA/D for multi-objective and many-objective optimization," Complex \& Intelligent Systems, vol. 3, no. 4, pp. 279294, 2017.

[27] M. Asafuddoula, T. Ray, and R. Sarker, "A decomposition based evolutionary algorithm for many objective optimization," IEEE Transactions on Evolutionary Computation, vol. 19, no. 3, pp. 445460, 2015.

[28] Y. Yuan, H. Xu, B. Wang, and X. Yao, "A new dominance relationbased evolutionary algorithm for many-objective optimization," IEEE Transactions on Evolutionary Computation, vol. 20, no. 1, pp. $16-37,2016$.

[29] A. G. Hernández-Díaz, L. V. Santana-Quintero, C. A. C. Coello, and J. Molina, "Pareto-adaptive $\epsilon$-dominance," Evolutionary computation, vol. 15, no. 4, pp. 493-517, 2007.

[30] S. Jiang, Z. Cai, J. Zhang, and Y.-S. Ong, "Multiobjective optimization by decomposition with Pareto-adaptive weight vectors," in Proceedings of the Seventh International Conference on Natural Computation, vol. 3, 2011, pp. 1260-1264.

[31] S. Z. Martínez, V. A. S. Hernández, H. Aguirre, K. Tanaka, and C. A. C. Coello, "Using a family of curves to approximate the Pareto front of a multi-objective optimization problem," in Proceedings of the 13th International Conference on Parallel Problem Solving from Nature, 2014, pp. 682-691.

[32] A. Zhou, Q. Zhang, and Y. Jin, "Approximating the set of Paretooptimal solutions in both the decision and objective spaces by an estimation of distribution algorithm," IEEE transactions on evolutionary computation, vol. 13, no. 5, pp. 1167-1189, 2009.

[33] K. Deb, L. Thiele, M. Laumanns, and E. Zitzler, Scalable test problems for evolutionary multiobjective optimization. Springer London, 2005.

[34] R. Wang, J. Xiong, H. Ishibuchi, G. Wu, and T. Zhang, "On the effect of reference point in MOEA/D for multi-objective optimization," Applied Soft Computing, vol. 58, pp. 25-34, 2017.

[35] C. S. Miranda and F. J. Von Zuben, "Necessary and sufficient conditions for surrogate functions of Pareto frontiers and their synthesis using gaussian processes," IEEE Transactions on Evolutionary Computation, vol. 21, no. 1, pp. 1-13, 2017.

[36] J. J. Moré, "The Levenberg-Marquardt algorithm: Implementation and theory," in Numerical analysis. Springer, 1978, pp. 105-116. 
[37] M. T. Hagan and M. B. Menhaj, "Training feedforward networks with the Marquardt algorithm," IEEE Transactions on Neural Networks, vol. 5, no. 6, pp. 989-993, 1994.

[38] X. Zhang, Y. Tian, R. Cheng, and Y. Jin, "A decision variable clustering-based evolutionary algorithm for large-scale manyobjective optimization," IEEE Transactions on Evolutionary Computation, vol. 22, no. 1, pp. 97-112, 2018.

[39] Y. Tian, H. Wang, X. Zhang, and Y. Jin, "Effectiveness and efficiency of non-dominated sorting for evolutionary multiand many-objective optimization," Complex $\mathcal{E}$ Intelligent Systems, vol. 3, no. 4, pp. 247-263, 2017.

[40] Y. Tian, R. Cheng, X. Zhang, and Y. Jin, "PlatEMO: A MATLAB platform for evolutionary multi-objective optimization," IEEE Computational Intelligence Magazine, vol. 12, no. 4, pp. 73-87, 2017.

[41] E. Zitzler and S. Künzli, "Indicator-based selection in multiobjective search," in Proceedings of the 8th International Conference on Parallel Problem Solving from Nature, 2004, pp. 832-842.

[42] K. Li, K. Deb, Q. Zhang, and S. Kwong, "Combining dominance and decomposition in evolutionary many-objective optimization," IEEE Transactions on Evolutionary Computation, vol. 19, no. 5, pp. 694-716, 2015.

[43] Y. Xiang, Z. Yuren, M. Li, and Z. Chen, "A vector angle based evolutionary algorithm for unconstrained many-objective optimization," IEEE Transactions on Evolutionary Computation, vol. 21, no. 1, pp. 131-152, 2017.

[44] H. Li, Q. Zhang, and J. Deng, "Biased multiobjective optimization and decomposition algorithm," IEEE Transactions on Cybernetics, vol. 47, no. 1, pp. 52-66, 2017.

[45] K. D. E. Zitzler and L. Thiele, "Comparison of multiobjective evolutionary algorithms: Empirical results," Evolutionary Computation, vol. 8, no. 2, pp. 173-195, 2000.

[46] L. B. S. Huband, P. Hingston and L. While, "A review of multiobjective test problems and a scalable test problem toolkit," IEEE Transactions on Evolutionary Computation, vol. 10, no. 5, pp. 477506, 2006

[47] K. Deb, Multi-Objective Optimization Using Evolutionary Algorithms. New York: Wiley, 2001.

[48] K. Deb and M. Goyal, "A combined genetic adaptive search (GeneAS) for engineering design," Computer Science and Informatics, vol. 26, no. 4, pp. 30-45, 1996.

[49] E. Zitzler, L. Thiele, M. Laumanns, C. M. Fonseca, and V. G. Da Fonseca, "Performance assessment of multiobjective optimizers: An analysis and review," IEEE Transactions on Evolutionary Computation, vol. 7, no. 2, pp. 117-132, 2003.

[50] Y. Tian, X. Xiang, X. Zhang, R. Cheng, and Y. Jin, "Sampling reference points on the Pareto fronts of benchmark multi-objective optimization problems," in Proceedings of the 2018 IEEE Congress on Evolutionary Computation, 2018, in press.

[51] D. A. Van Veldhuizen, "Multiobjective evolutionary algorithms: Classifications, analyses, and new innovations," Ph.D. dissertation, Department of Electrical and Computer Engineering, Graduate School of Engineering, Air Force Institute of Technology, Ohio, USA, 1999.

[52] R. Cheng, Y. Jin, M. Olhofer, and B. Sendhoff, "Test problems for large-scale multiobjective and many-objective optimization," IEEE Transactions on Cybernetics, vol. 47, no. 12, pp. 4108-4121, 2017.

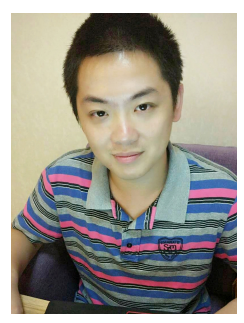

Ye Tian received the B.Sc., M.Sc., and Ph.D. degrees from Anhui University, Hefei, China, in 2012, 2015, and 2018, respectively.

He is currently a Lecturer with the Institute of Physical Science and Information Technology, Anhui University, Hefei, China. His current research interests include multi-objective optimization methods and their application. He is the recipient of the 2018 IEEE Transactions on Evolutionary Computation Outstanding Paper Award.

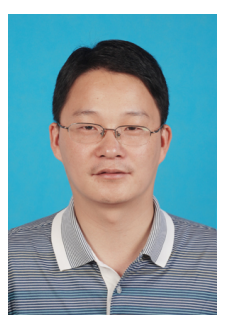

Xingyi Zhang received the B.Sc. degree from Fuyang Normal College, Fuyang, China, in 2003, and the M.Sc. and Ph.D. degrees from Huazhong University of Science and Technology, Wuhan, China, in 2006 and 2009, respectively.

He is currently a Professor with the School of Computer Science and Technology, Anhui University, Hefei, China. His current research interests include unconventional models and algorithms of computation, multi-objective optimization, and membrane computing. He is the recipient of the 2018 IEEE Transactions on Evolutionary Computation Outstanding Paper Award.

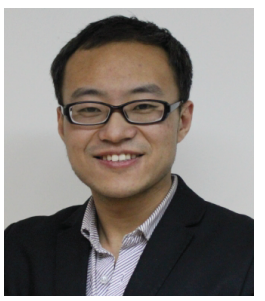

Ran Cheng (M'16) received the B.Sc. degree from the Northeastern University, Shenyang, China, in 2010, and the Ph.D. degree from the University of Surrey, Guildford, U.K., in 2016

He is currently an Assistant Professor with the Department of Computer Science and Engineering, Southern University of Science and Technology, Shenzhen, China. His current research interests include evolutionary multiobjective optimization, model-based evolutionary algorithms, large-scale optimization, swarm intelligence, and deep learning. He is the founding chair of IEEE Symposium on Model Based Evolutionary Algorithms (IEEE MBEA). He is the recipient of the 2018 IEEE Transactions on Evolutionary Computation Outstanding Paper Award, and the 2019 IEEE Computational Intelligence Society (CIS) Outstanding Ph.D. Dissertation Award.

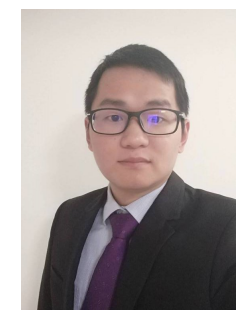

Cheng $\mathrm{He}$ received the B.Eng. degree from Wuhan University of Science and Technology, Wuhan, China, in 2012, and the Ph.D. degree from Huazhong University of Science and Technology, Wuhan, China, in 2018.

He is currently a Research Fellow with the Department of Computer Science and Engineering, Southern University of Science and Technology, Shenzhen, China. His current research interests include mutli-/many-objective optimization, large-scale optimization, surrogateassisted evolutionary optimization, and the detection of power transformer problems.

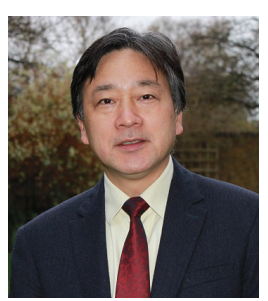

Yaochu Jin (M'98-SM'02-F'16) received the B.Sc., M.Sc., and Ph.D. degrees from Zhejiang University, Hangzhou, China, in 1988, 1991, and 1996, respectively, and the Dr.-Ing. degree from Ruhr University Bochum, Bochum, Germany, in 2001

He is currently a Professor in Computational Intelligence, Department of Computer Science, University of Surrey, Guildford, U.K., where he heads the Nature Inspired Computing and Engineering Group. He is also affiliated with the Department of Computer Science and Technology, Taiyuan University of Science and Technology, Taiyuan 030024, China. He is a Finland Distinguished Professor and a Changiiang Distinguished Visiting Professor appointed. He has (co)authored over 200 peer-reviewed journal and conference papers and been granted eight patents on evolutionary optimization.

He is the Editor-in-Chief of the IEEE Transactions on Cognitive and Developmental Systems and Complex \& Intelligent Systems. He is also an Associate Editor or Editorial Board Member of the IEEE Transactions on Evolutionary Computation, IEEE Transactions on Cybernetics, IEEE Transactions on Nanobioscience, Evolutionary Computation, BioSystems, Soft Computing, and Natural Computing. He is an IEEE Distinguished Lecturer (2017-2019). He is the recipient of the 2014 and 2016 IEEE Computational Intelligence Magazine Outstanding Paper Award, the 2018 IEEE Transactions on Evolutionary Computation Outstanding Paper Award, and the Best Paper Award of the 2010 IEEE Symposium on Computational Intelligence in Bioinformatics and Computational Biology. He is a Fellow of IEEE. 